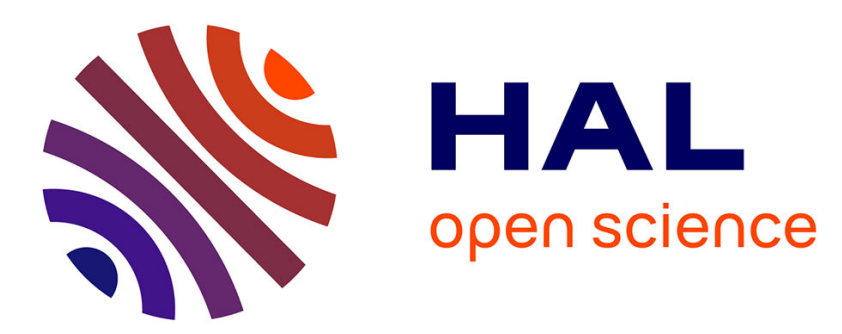

\title{
Daclatasvir Prevents Hepatitis C Virus Infectivity by Blocking Transfer of Viral Genome to Assembly Sites.
}

Bertrand Boson, Solène Denolly, Fanny Turlure, Christophe Chamot, Marlène Dreux, Cosset François-Loïc

\section{- To cite this version:}

Bertrand Boson, Solène Denolly, Fanny Turlure, Christophe Chamot, Marlène Dreux, et al.. Daclatasvir Prevents Hepatitis C Virus Infectivity by Blocking Transfer of Viral Genome to Assembly Sites.. Gastroenterology, 2016, 152 (4), pp.895-907. 10.1053/j.gastro.2016.11.047 . inserm$01425689 \mathrm{v} 2$

\author{
HAL Id: inserm-01425689 \\ https://www.hal.inserm.fr/inserm-01425689v2
}

Submitted on 6 Mar 2017

HAL is a multi-disciplinary open access archive for the deposit and dissemination of scientific research documents, whether they are published or not. The documents may come from teaching and research institutions in France or abroad, or from public or private research centers.
L'archive ouverte pluridisciplinaire HAL, est destinée au dépôt et à la diffusion de documents scientifiques de niveau recherche, publiés ou non, émanant des établissements d'enseignement et de recherche français ou étrangers, des laboratoires publics ou privés. 


\title{
Daclatasvir Prevents Hepatitis C Virus Infectivity by Blocking Transfer of the Viral Genome to Assembly Sites
}

\author{
Bertrand Boson, ${ }^{1}$ Solène Denolly, ${ }^{1}$ Fanny Turlure, ${ }^{1}$ Christophe Chamot, ${ }^{2}$ \\ Marlène Dreux, ${ }^{1}$ and François-Loïc Cosset $^{1}$
}

${ }^{1} \mathrm{CIRI}$ - International Center for Infectiology Research, Team EVIR, Inserm, U1111, Université Claude Bernard Lyon 1, CNRS, UMR5308, Ecole Normale Supérieure de Lyon, Univ Lyon, F-69007, Lyon, France; and ${ }^{2}$ Plateau Technique Imagerie/ Microcopie, Lyon Bio Image, SFR-BioSciences, ENS de Lyon, Inserm US8, CNRS UMS3444, UCBL, France

BACKGROUND \& AIMS: Daclatasvir is a direct-acting antiviral agent and potent inhibitor of NS5A, which is involved in replication of the hepatitis $\mathrm{C}$ virus (HCV) genome, presumably via membranous web shaping, and assembly of new virions, likely via transfer of the HCV RNA genome to viral particle assembly sites. Daclatasvir inhibits the formation of new membranous web structures and, ultimately, of replication complex vesicles, but also inhibits an early assembly step. We investigated the relationship between daclatasvir-induced clustering of $\mathrm{HCV}$ proteins, intracellular localization of viral RNAs, and inhibition of viral particle assembly. METHODS: Cell-culture-derived HCV particles were produced from Huh7.5 hepatocarcinoma cells in presence of daclatasvir for short time periods. Infectivity and production of physical particles were quantified and producer cells were subjected to subcellular fractionation. Intracellular colocalization between core, E2, NS5A, NS4B proteins, and viral RNAs was quantitatively analyzed by confocal microscopy and by structured illumination microscopy. RESULTS: Short exposure of HCVinfected cells to daclatasvir reduced viral assembly and induced clustering of structural proteins with non-structural HCV proteins, including core, E2, NS4B, and NS5A. These clustered structures appeared to be inactive assembly platforms, likely owing to loss of functional connection with replication complexes. Daclatasvir greatly reduced delivery of viral genomes to these core clusters without altering HCV RNA colocalization with NS5A. In contrast, daclatasvir neither induced clustered structures nor inhibited HCV assembly in cells infected with a daclatasvir-resistant mutant (NS5A-Y93H), indicating that daclatasvir targets a mutual, specific function of NS5A inhibiting both processes. CONCLUSIONS: In addition to inhibiting replication complex biogenesis, daclatasvir prevents viral assembly by blocking transfer of the viral genome to assembly sites. This leads to clustering of HCV proteins because viral particles and replication complex vesicles cannot form or egress. This dual mode of action of daclatasvir could explain its efficacy in blocking HCV replication in cultured cells and in treatment of patients with HCV infection.

Keywords: Chronic Hepatitis C; Direct-Acting Antiviral Agent; DAA.

$\mathrm{H}$ epatitis $\mathrm{C}$ virus (HCV) infection is a leading cause of chronic liver diseases worldwide. With 180 million people persistently infected, chronic HCV infection, which induces end-stage liver diseases such as liver cirrhosis and hepatocellular carcinoma, presents a public health problem of high socioeconomic impact. No protective vaccine exists against HCV. However, the development of direct-acting antivirals targeting different proteins and functions in HCV life cycle has dramatically changed the treatment options for chronic hepatitis $\mathrm{C}$, leading to new hopes to cure HCV.

$\mathrm{HCV}$ has a positive-sense single-strand RNA (RNA[+]) genome encoding viral proteins, including an assembly module (C-non-structural [NS] 2) encompassing the capsid protein (core); E1 and E2 surface glycoproteins that are incorporated in viral particles; the p7 viroporin and NS2 protein that support virion assembly; and a replication module encompassing the NS proteins NS3, NS4A, NS4B, NS5A, and NS5B, which are sufficient to support viral RNA replication and also contribute to virion formation through an unclear process.

The recent development of direct-acting antiviral has benefited greatly from discoveries in HCV molecular virology and host-virus interactions. ${ }^{1}$ The primary targets of direct-acting antiviral ${ }^{2}$ include the serine protease activity of NS3/NS4A, the multifunctional RNA-binding phosphoprotein NS5A, and the RNA-dependent RNA polymerase NS5B. Daclatasvir (DCV) and DCV-related molecules were discovered through screening of compounds inhibiting HCV subgenomic replicons. ${ }^{3}$ In addition to being effective inhibitors of HCV replication, with $50 \%$ effective concentration values in the pM range, recent studies have reported that DCV acts on a distinct stage of replication, namely viral particle assembly. ${ }^{4-6}$ Exposure of cell-culture-derived HCV infected cells to DCV for short time periods decreased production of infectious particles before decay of intracellular HCV RNA and polyprotein levels.

Although DCV's mode of action is unclear, it specifically targets NS5A, ${ }^{3,7}$ particularly its domain I (DI), which has functions associated with genome replication, ${ }^{8}$ in line with identified DCV-resistant mutations in DI (eg, L31 and Y93). ${ }^{3}$

Abbreviations used in this paper: $\mathrm{CHX}$, cycloheximide; DCV, daclatasvir DI, domain I; DMV, double membrane vesicle; ER, endoplasmic reticulum; HCV, hepatitis C virus; LD, lipid droplet; MOI, multiplicity of infection; NS, non-structural protein; PBS, phosphate-buffered saline; RC, replication complex; SIM, structured illumination microscopy.

(Q) Most current article

(C) 2017 by the AGA Institute 0016-5085/\$36.00

http://dx.doi.org/10.1053/j.gastro.2016.11.047 
Importantly, DCV inhibits formation of double-membrane vesicles (DMVs) that contain the HCV RNA replication complex (RC) $)^{6,9}$ and are induced by non-structural proteins, particularly NS4B and NS5A. ${ }^{10,11}$ In addition, other studies reported that DCV or DCV-related compounds decrease mobility, $^{12}$ intracellular redistribution, ${ }^{13,14}$ and/or clustering ${ }^{5,12,15}$ of NS5A. Despite these remarkable findings, there is no clear consensus on the role, location, and kinetics of these events, possibly because of differences in experimental conditions. NS5A is an HCV RNA-binding protein ${ }^{16,17}$ that mediates HCV RNA transfer to viral particles, ${ }^{18}$ so it seems possible that altered NS5A intracellular localization and/or trafficking could prevent assembly of infectious particles.

We investigated the relationship between DCV-induced clustering of HCV proteins and inhibition of viral particle assembly. We studied the intracellular localization of HCV assembly and RC proteins along with $\operatorname{HCV} \mathrm{RNA}(+)$ and $\mathrm{RNA}(-)$ species within HCV-infected cells at early time points after DCV exposure and compared these events with the production of infectious particles. We show that in addition to inhibiting DMV biogenesis, DCV prevents HCV assembly by blocking the transfer of viral genomes to virion assembly proteins. This leads to clustering of HCV proteins, likely because viral particles and RC vesicles cannot form and/or egress from the endoplasmic reticulum (ER) membranes. This dual mode of action of DCV could account for its extremely high potency in cell culture and, most likely, in patients.

\section{Materials and Methods}

\section{Cell Culture and Reagents}

Huh7.5 cells were grown in Dulbecco's modified minimal essential medium (Invitrogen, Cergy-Pontoise, France) supplemented with $100 \mathrm{U} / \mathrm{mL}$ penicillin, $100 \mu \mathrm{g} / \mathrm{mL}$ streptomycin, and $10 \%$ fetal bovine serum.

\section{Expression Constructs}

pFK-JFH1wt_dg, pFK-JFH1/J6/C-846_dg and pFK-Jc1Y93H_dg plasmids ${ }^{6,19}$ were kind gifts from R. Bartenschlager.

\section{Cell-Culture-Derived Hepatitis C Virus Production and Titration}

Cell-culture-derived HCV production procedures were described previously. ${ }^{20}$ Supernatants infectivity titers were determined as focus-forming units per milliliter. Serial dilutions of supernatants were used to infect Huh7.5 cells, focus-forming units were determined 3 days post-infection by counting NS5A-immunostained foci.

\section{Immunofluorescence and Confocal Microscopy Imaging}

Huh7.5 cells grown on uncoated 14-mm-diameter glass coverslips were infected at multiplicity of infection (MOI) of 0.2. At indicated times post-infection, cells were washed with phosphate-buffered saline (PBS), fixed with 3\% paraformaldehyde in PBS for 15 minutes, quenched with $50 \mathrm{mM}$ $\mathrm{NH}_{4} \mathrm{Cl}$, and permeabilized with $0.1 \%$ Triton $\mathrm{X}-100$ for 7 minutes. Fixed cells were then incubated for 1 hour with primary antibodies in $1 \%$ bovine serum albumin/PBS, washed and stained for 1 hour with the corresponding fluorescent Alexa-conjugated secondary antibody (Alexa-488, Alexa-555, and Alexa-647, Molecular Probes Europe BV, Leiden, The Netherlands) in 1\% bovine serum albumin/PBS. Lipid droplets (LDs) were stained with $10 \mu \mathrm{g} / \mathrm{mL}$ Bodipy 493/503 (Molecular Probes) according to the manufacturer's instructions. Cells were washed 3 times with PBS, stained for nuclei with Hoechst (Molecular Probes) for 5 minutes when stated, washed, and mounted in Mowiol (Fluka, Buchs, Switzerland) before image acquisition with LSM-510 or LSM-710 confocal microscopes.

\section{Combined Detection of Hepatitis C Virus RNA by Fluorescent In Situ Hybridization and Viral Proteins}

Viral proteins were first immunostained as described. After a post-fixation step with 3\% paraformaldehyde for 15 minutes and 3 washes with PBS, HCV RNA(+), and $\operatorname{RNA}(-)$ strands were detected using probe sets that target regions between nucleotide positions 3733-4870 and 4904-5911, respectively, in the JFH1 genome using QuantiGene ViewRNA ISH Cell Assay kit (Panomics/Affymetrix, Santa Clara, CA) according to the manufacturer's instructions, except for the protease digestion step that was omitted. Omission of protease digestion step did not affect detection of HCV RNA(+) or RNA(-) foci (data not shown). Nuclei staining, slide mounting, and acquisition were performed as described.

\section{Structured Illumination Microscopy}

Huh7.5 cells were grown on high precision cover glasses and infected (MOI $=0.2$ ). At 72 hours post-infection, cells were fixed with $3 \%$ paraformaldehyde and stained as described. Cells were then mounted in Fluoromount-G (Southern Biotech, Birmingham, AL) before image acquisition with Elyra PS-1 microscope (Zeiss, Oberkochen, Germany). Images were reconstructed using the Zen 2012 Black software (Zeiss).

\section{Image Analysis and Quantifications}

Images were analyzed with the ImageJ software (imagj.nih. gov). For quantifications of structures, colocalized pixels were extracted with the ColocalizeRGB plugin with auto-thresholding and a pixel ratio between paired channels set to $50 \%$. They were then segmented with the Watershed algorithm and quantified with the Analyze Particles function of ImageJ. Only structures with size $>0.02 \mu \mathrm{m}^{2}$, which corresponds to half of the resolution limit of confocal laser scanning microscopy, were recorded. For quantification of structures apposed at the edge of LDs, an ImageJ macro was developed (for details, see Supplementary Results) to search for variation of max intensity in the neighborhood of each LD as an indicator of proximity. Structures distant from LDs by $>3$ pixels (approximately 205-207 nm) were considered as non-apposed at the edge of LDs. When stated, the Pearson's correlation coefficients were calculated by using the JACoP plugin.

\section{Statistical Analysis}

Significance values were calculated by applying the 2-tailed, unpaired Mann-Whitney test for image analyses and the paired $t$ test for the quantification of viral RNA, core protein, 
and infection assays, using the GraphPad Prism 6 software (GraphPad Software, La Jolla, CA). $P$ values $<.05$ were considered statistically significant and the following denotations were used: ${ }^{* * * *} P \leq .0001 ;{ }^{* * *} P \leq .001 ;{ }^{* *} P \leq .01 ;{ }^{*} P \leq .05$; ns (not significant) $\bar{P}>.05$.

\section{Results}

\section{An NS5A Inhibitor Preventing Viral Assembly Induces Co-Clustering of Structural and Non-Structural Proteins}

To understand the impact of DCV on viral particle assembly, first we confirmed and extended previous studies ${ }^{4,5}$ indicating that, unlike the DCV-resistant NS5A-mutant Jc1Y93H virus, ${ }^{6}$ a 6-hour-long treatment with $1 \mathrm{nM}$ DCV had no effect on intracellular HCV RNA (Supplementary Figure $1 A$, left panels) and core protein (Supplementary Figure $1 A$, right panel) levels in cells infected with Jc1 virus. In contrast, a longer treatment ( 24 hours) reduced Jc1 intracellular RNAs by $>10$-fold, in accordance with these former reports. As reported previously, ${ }^{4,5}$ inhibition of infectivity was detectable from 2 hours after the initiation of DCV treatment (Supplementary Figure $2 A$ and $B$ ). Upon a 6-hour-long treatment, the levels of both intracellular and extracellular infectious particles were reduced by approximately 13-fold and 4-fold, respectively, whereas Jc1-Y93H virus infectivity was not affected (Supplementary Figures $1 B$ and $2 A$ and $B$ ). Finally, we found that DCV inhibited the production of physical particles (Supplementary Figure 1C), as judged by the inhibition of both secreted viral RNAs and core protein from infected cells. Altogether, these results indicated that the decrease of viral production via short-time DCV treatment is due to inhibition of viral assembly rather than egress of particles, whereas longer treatments inhibit both assembly and replication.

Because DCV alters NS5A intracellular distribution ${ }^{5,12-15}$ and core colocalizes with several other HCV proteins in Jc1infected cells, including E2, NS2, NS3, NS4B, and NS5A (Supplementary Figure 3), we next wondered whether DCV inhibits viral assembly by altering the association of structural proteins with non-structural proteins from RCs. We investigated by confocal microscopy the effect of short-time DCV treatments on HCV proteins that mark either component of viral particles ${ }^{20}$ - core and $\mathrm{E} 2$ or $\mathrm{RCs}^{10,21}$ - that is, NS5A (Figure 1) and NS4B (Figure 2). Importantly, punctate
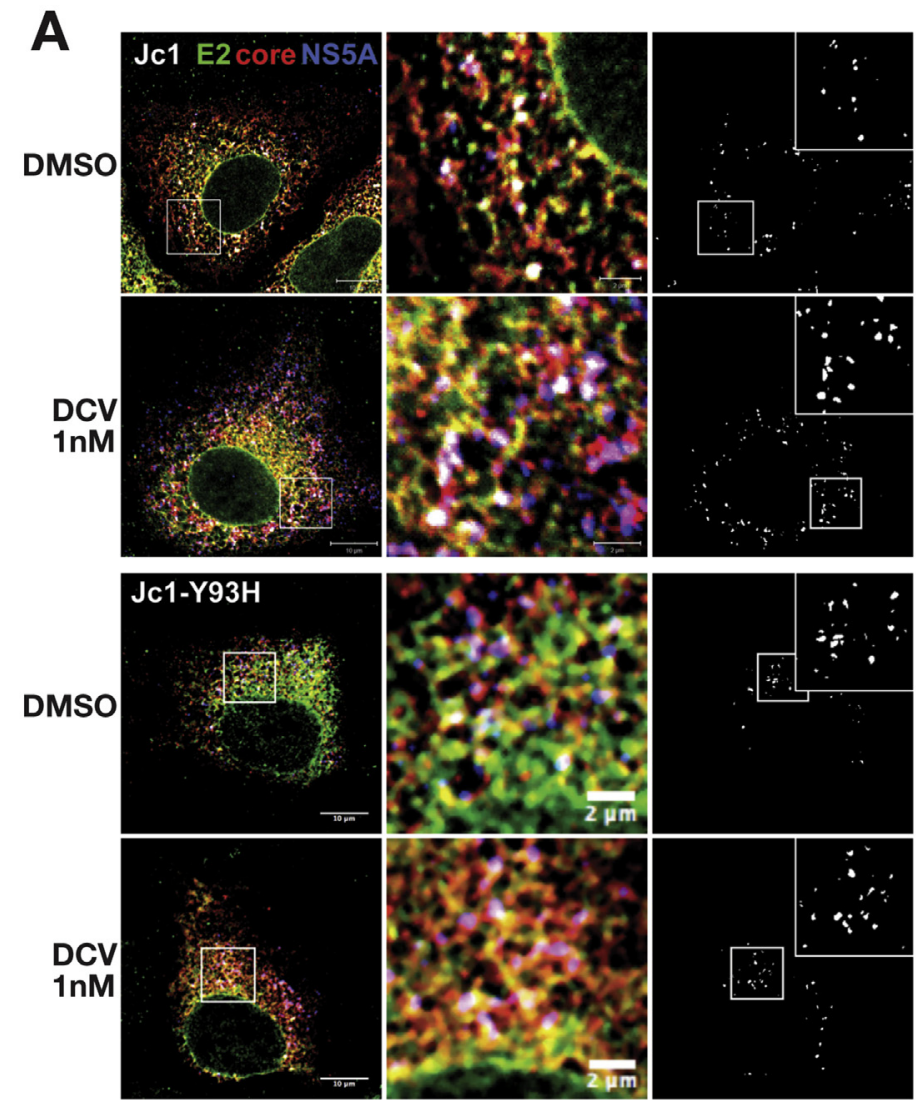

B
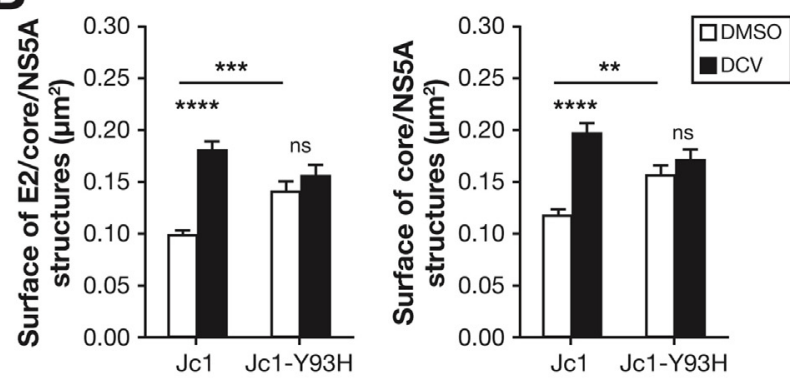

C
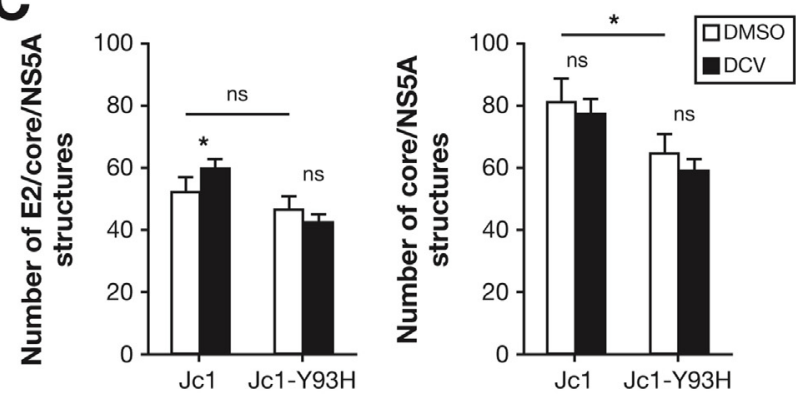

Figure 1. DCV induces the clustering of E2/core/NS5A structures. Huh7.5 cells infected with Jc1 or Jc1-Y93H viruses $(\mathrm{MOI}=0.2)$ were incubated at 66 hours post-infection with $1 \mathrm{nM} \mathrm{DCV}$ or dimethyl sulfoxide (DMSO) for 6 hours. After staining for HCV E2, core, and NS5A proteins, colocalization of core (red channel) with E2 (green channel) and NS5A (blue channel) proteins was analyzed by confocal microscopy $(A)$. Scale bars of panels and zooms from squared area represent $10 \mu \mathrm{m}$ and $2 \mu \mathrm{m}$, respectively. Colocalized pixels (white channel) between red, green, and blue channels were extracted with the ColocalizeRGB plugin of ImageJ. The surface $(B)$ and number $(C)$ of E2/core/NS5A and core/NS5A structures were quantified with ImageJ. For each condition, $30-50$ cells were quantified. ${ }^{\star \star \star \star} P \leq .0001 ;{ }^{\star \star \star} P \leq .001 ;{ }^{\star \star} P \leq .01 ;{ }^{\star} P \leq .05$; ns (not significant) $P>.05$. 


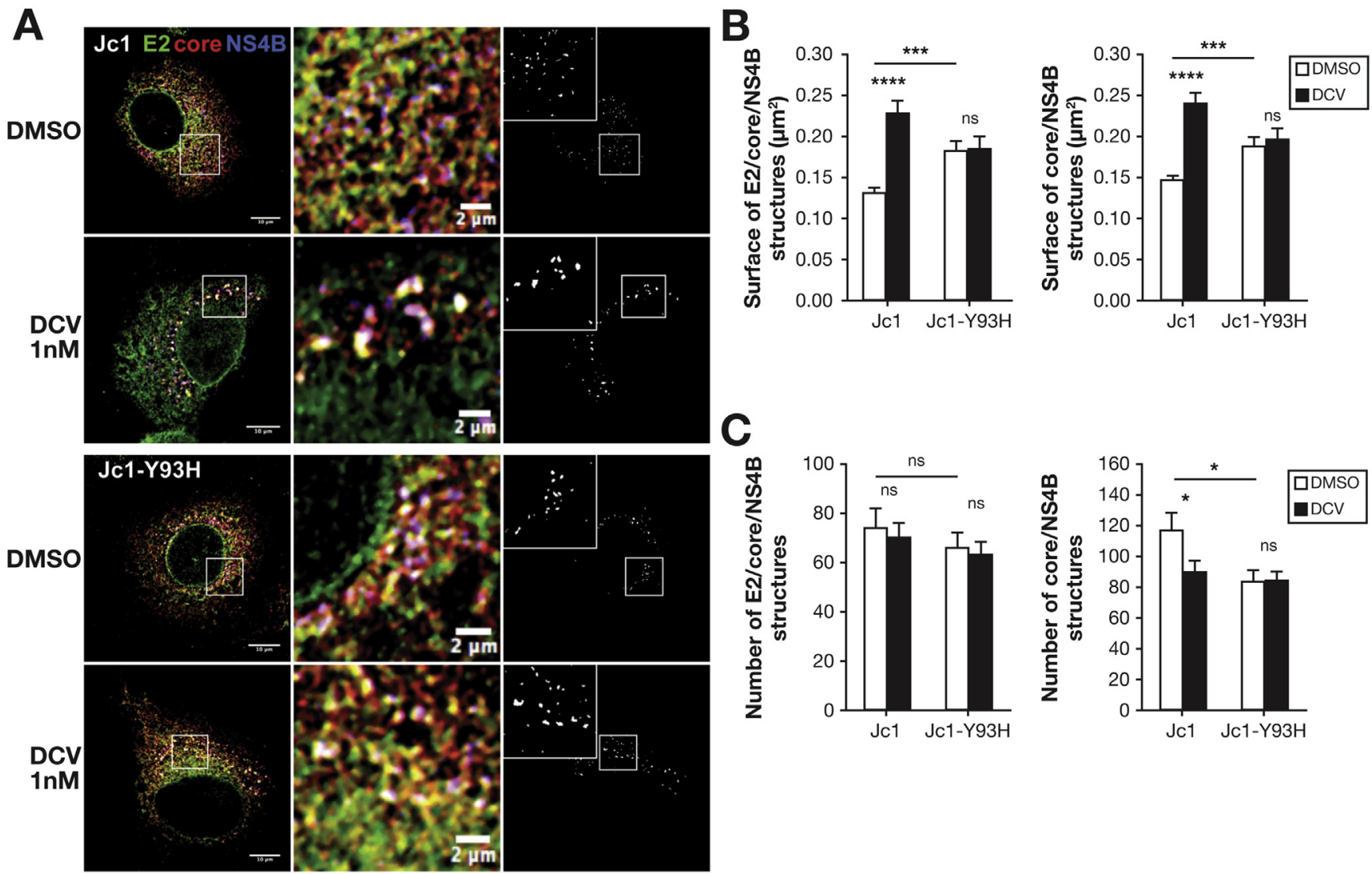

Figure 2. DCV induces the clustering of E2/core/NS4B structures. Cells were infected, treated, and analyzed as in Figure 1. Confocal analysis of core with E2 and NS4B, displayed as in Figure 1A, with NS4B instead of NS5A in the blue channel ( $A$ ). The surface $(B)$ and number $(C)$ of E2/core/NS4B and core/NS4B structures were quantified with ImageJ. DMSO, dimethyl sulfoxide. ${ }^{\star \star \star \star} P \leq .0001 ;{ }^{\star \star \star} P \leq .001 ;{ }^{*} P \leq .05$; ns (not significant) $P>.05$.

structures colocalizing E2, core, and NS5A proteins were readily detected in Jc1-infected cells at 72 hours postinfection (see the extractions of E2/core/NS5A colocalization pixels in the right panels $[A]$ of either Figure 1 or 2 and quantifications in panels $B-C$ ). Strikingly, after a 6-hour-long DCV treatment, they appeared approximately $85 \%$ bigger than without treatment (Figure $1 B$ ). Likewise, E2/core/NS4B punctate structures were also detected in Jc1-infected cells (Figure 2); yet, similar to E2/core/NS5A structures, their size steadily increased from 2 hours after DCV treatment (Supplementary Figure 2C) and was approximately $75 \%$ bigger than without DCV treatment after a 6-hour-long incubation (Figure 2B), although their number remained constant (Supplementary Figure 2D, Figure $2 C$ ). Of note, short-time DCV treatments from 18 hours post-infection also induced strong enlargements of both E2/core/NS5A and E2/core/NS4B punctate structures, by up to 3-fold (data not shown). These core/NS5A and core/NS4B structures clustering with E2 (Figures 1 and 2) likely gathered within combined structures, as indicated by the enlargement of punctae that included core, NS5A, and NS4B upon short-time DCV treatments (Supplementary Figure 4). Importantly, the Y93H DCV-resistant mutation abolished DCV-induced enlargement of all these structures (Figures 1 and 2, Supplementary Figure $2 C-D$ ), indicating that their alteration likely involved DCV effect on NS5A.
Finally, DCV treatment blocked NS5A hyperphosphorylation, as reported by others, ${ }^{22,23}$ which correlated with the co-clustering of structural and non-structural proteins and with the concomitant decrease of assembly of infectious viral particles (Supplementary Figures 5 and 2). As noted by others, ${ }^{6,22}$ the Y93H DCV-resistant mutation altered DCV-mediated inhibition of NS5A hyperphosphorylation (Supplementary Figure 5). As NS5A hyper-phosphorylation modulates NS5A functions in both replication and assembly, ${ }^{8}$ these results further implied that DCV targeting of NS5A DI domain function(s) is pivotal for both steps.

Altogether, these results suggested that DCV acts shortly after administration to block viral assembly and results in the co-clustering of structural proteins with RC components.

\section{In Absence of Daclatasvir Treatment, Structural and Nonstructural Proteins Colocalize Over Time Post-Infection Within Accruing Punctate Structures}

Our results suggested that, rather than inducing novel structures, DCV expands or merges pre-existing structures colocalizing structural and nonstructural proteins. Accordingly, DCV did not modify the cellular localization and membrane association of E2, core, NS4B, and NS5A proteins, 
as indicated by subcellular fractionation assays of infected cells that did not reveal changes in their fraction contents upon DCV treatment (Supplementary Figure 6A). Altogether, this prompted us to characterize such pre-existing structures, aiming at understanding the mode of action of DCV on HCV assembly inhibition.

Using super-resolution microscopy (3-dimensional structured illumination microscopy), we found that E2/ core/NS4B dot structures gather NS4B part(s) and E2-dense parts, with core joining either areas (Figure $3 A$ ). Such structures might represent territories connecting the sites of replication and assembly. Therefore, to investigate their formation, they were characterized over time post-infection with the Jc1 virus comparatively to the JFH1 virus that produces 50-100-fold less infectious viral particles ${ }^{20}$ despite identical replication rates. ${ }^{19}$ At early time points post-infection (before 24 hours), core and NS4B appeared as punctate structures, whereas E2 was spread throughout the ER (Figure 4A, Supplementary Figure 7). Few dots of colocalization between core, E2, and NS4B were detected at early time points in both Jc1- and JFH1-infected cells; yet, their number increased subsequently. Specifically, from 24 hours to 72 hours post-infection, the number and size of E2/ core/NS4B structures increased in Jc1-infected cells by approximately 20 -fold and 3-fold, respectively (Figure 4B,C). This suggested that core progressively concentrates in areas including E2 and/or NS4B, and that the structures containing both structural proteins and components of RCs appear concomitantly. Comparatively, the number and size of E2/core/NS4B structures increased slowly in JFH1
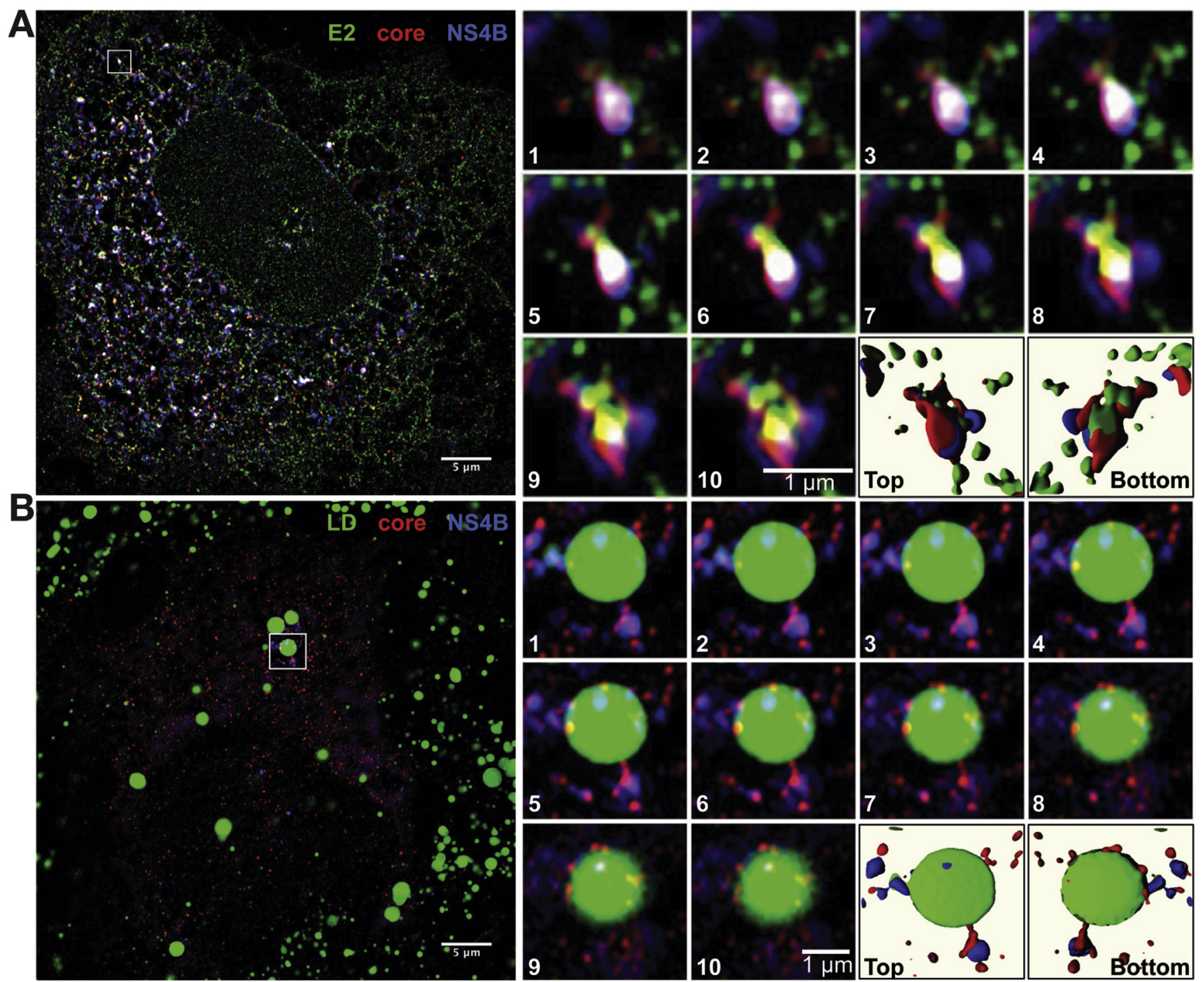

Figure 3. Three-dimensional structured illumination microscopy (3D-SIM) super-resolution imaging of E2/core/NS4B structures. Cells were infected with Jc1 virus (MOI =0.2), treated for 24 hours with $20 \mu \mathrm{g} / \mathrm{mL} \mathrm{CHX}$ at 48 hours post-infection, and fixed at 72 hours post-infection. Cells were stained for HCV E2, core, and NS4B proteins and for LDs. Colocalization of core (red channel) with E2 (green channel) and NS4B (blue channel) ( $A$ ) or core with LDs (green channel) and NS4B (blue channel) (B) was analyzed by 3D-SIM. Zooms of typical structures are represented as individual stack from top to bottom (referred to as $1-10)$ and 3D reconstructions from Imaris software are shown. Scale bars of panels and zooms from squared area represent 5 $\mu \mathrm{m}$ and $1 \mu \mathrm{m}$, respectively. 
A

Hours post-infection
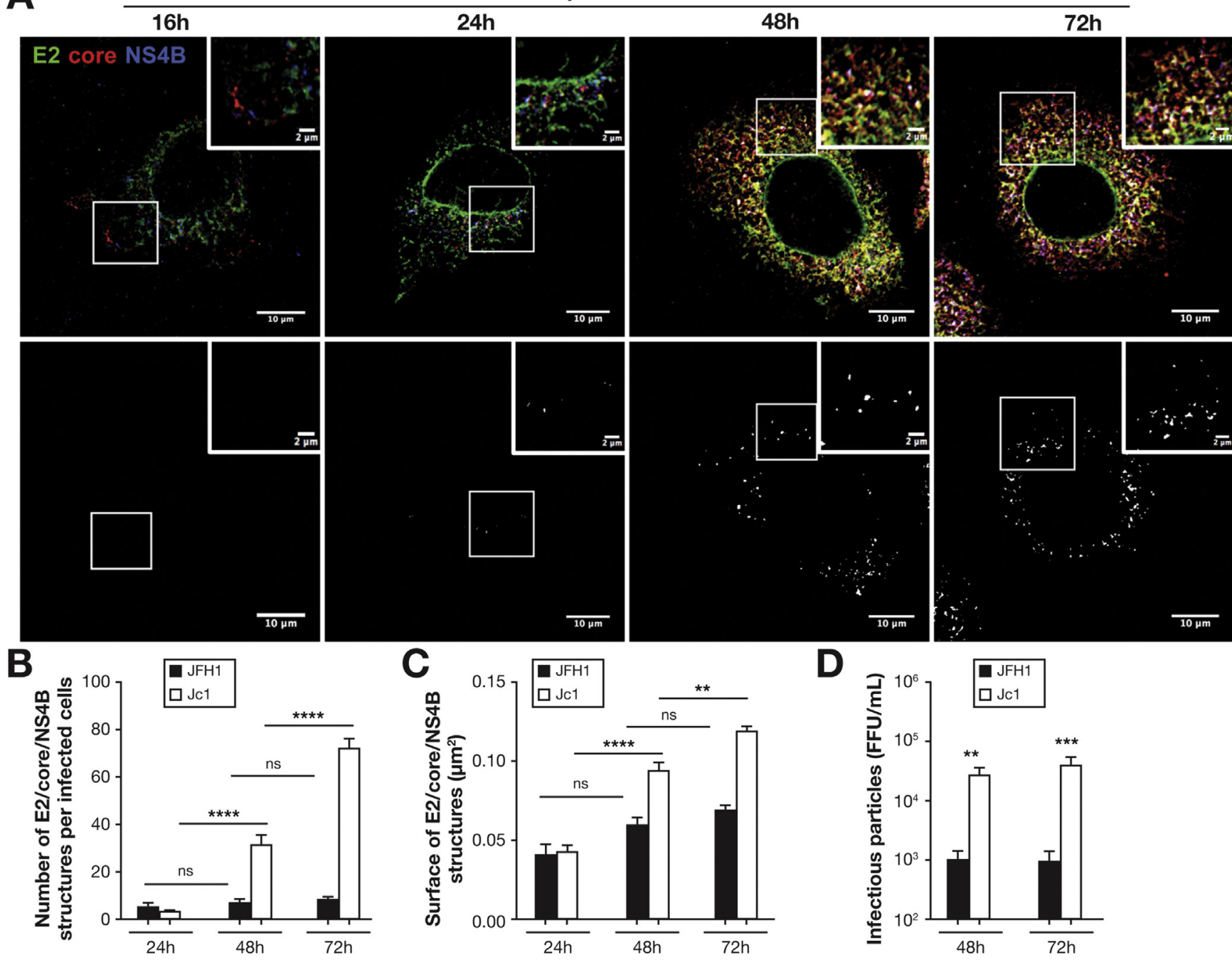

Figure 4. Structural proteins and NS4B colocalize within discrete structures. Cells infected with Jc1 or JFH1 viruses $(\mathrm{MOI}=0.2)$ were fixed at the indicated time post-infection and were then stained for HCV E2, core, and NS4B proteins. The colocalization of core (red channel) with E2 (green channel) and NS4B (blue channel) was analyzed by confocal microscopy. A representative picture of $\mathrm{Jc} 1$-infected cells is presented $(A)$. Scale bars of panels and zooms from squared area represent 10 $\mu \mathrm{m}$ and $2 \mu \mathrm{m}$, respectively. Colocalized pixels (white channel) between red, green, and blue channels were extracted with the ColocalizeRGB plugin of ImageJ (second row). The number $(B)$ and size $(C)$ of E2/core/NS4B structures were quantified with ImageJ. For each condition, 30-50 cells were quantified. The supernatants of infected cells were harvested at the indicated time post-infection and used to infect naïve Huh7.5 cells to determine virus infectivity $(D) .{ }^{\star \star \star \star} P \leq .0001 ;{ }^{\star \star \star} P \leq .001 ;{ }^{* \star} P \leq .01$; ns (not significant) $P>.05$.

virus-infected cells from 24 hours post-infection and were approximately 10 -fold less frequent at 72 hours postinfection as compared to Jc1 (Figure 4B), in agreement with the lower production of infectious viral particles (Figure $4 D$ ). The appearance of E2/core/NS4B structures, which correlated with infectivity levels, suggested that they could represent RCs connected to viral particle assembly components or sites.

\section{Daclatasvir Does Not Alter the Localization of Punctate Structures That Are Apposed to, But Not Associated With Lipid Droplets}

Previous studies reported a differential accumulation of HCV core in 2 distinct intracellular areas in Jc1- vs
JFH1-infected cells, that is, at the ER vs LDs, ${ }^{20,24}$ respectively, which may reflect the dissimilar capacity of core from either virus to engage in assembly. Thus, aiming at better characterizing the initiation of HCV assembly, we compared core localization at different time points post-infection. At 16 hours post-infection, the core protein showed intracellular localization patterns as small and discrete punctae that were distributed uniformly (Supplementary Figure 8A). These structures were still observed at 24 hours post-infection for both viruses; yet, in JFH1-infected cells, core also became detectable at the surface of LDs. At later time points, from 48 hours post-infection, the core protein was found clearly redistributed following 2 different patterns, that is, as a reticular pattern in Jc1-infected cells vs surrounding LDs in JFH1-infected cells (Supplementary Figure 8A). 
To determine how the initial core punctae were progressively redistributed in either pattern, we inhibited protein synthesis at 24 hours vs 48 hours post-infection by treating infected cells with cycloheximide (CHX) for 24 hours (Supplementary Figure 8A). After CHX treatment, the core protein of JFH1 virus was still observed as ring-like structures that completely surrounded $>95 \%$ of the LDs, suggesting that the core protein initially present as discrete punctae progressively reached and accumulated at LD surface. In sharp contrast, the early-detected discrete core punctae persisted in CHX-treated Jc1-infected cells and enlarged by approximately $50 \%$ from 48 hours to 72 hours post-infection (Supplementary Figure $8 A$ and $B$ ).

When we determined the localization of these core punctae in Jc1-infected cells, we found that they were not randomly distributed throughout the ER but rather, that a significant part, of approximately $40 \%$, was apposed at the edge of LDs (Figure $5 A$ and $B$ ), which represent specific sites where LDs are juxtaposed to the ER membrane and may gain their proteins. ${ }^{25}$ As about $10 \%$ of LDs in Jc1-infected cells were surrounded by core protein compared to $>95 \%$ in JFH1-infected cells (Figure 5C), this suggested that coreapposition at LD edges, rather than LD surface-association, promotes HCV early assembly steps. These findings implied that the core protein of Jc1 virus was not mobilized to or from the LD surface, but rather, was targeted and/or

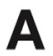

Core associated with LDs, on ER membrane

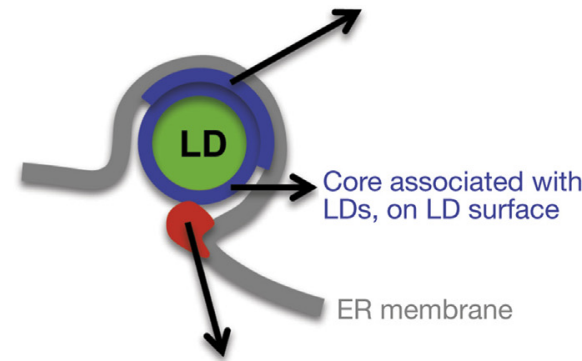

Core apposed at the edge of LDs, on ER membrane

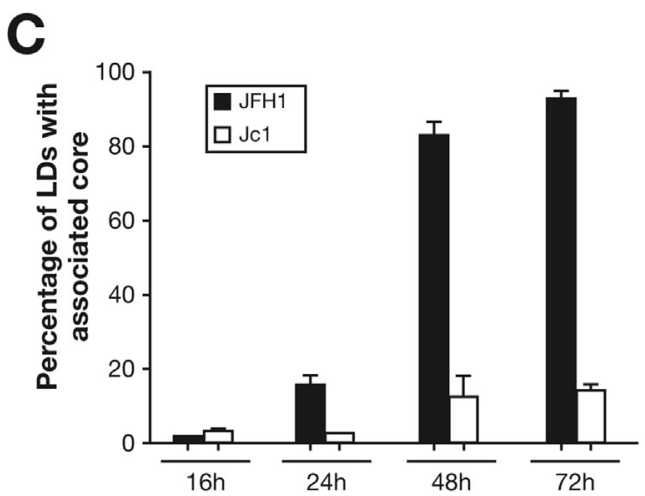

retained at ER membrane sites apposed to LDs. This also indicated that the reticular pattern detected at late time points post-infection in CHX-untreated cells reflected the progressive accrual and/or enlargement of core punctae (Supplementary Figure 8A). Altogether, these results suggested that the association of core on LD surface does not contribute to virion assembly, at least in a direct manner, and that, over time post-infection, the core protein of the highly infectious Jc1 virus progressively concentrates in large, LD edge-apposed punctate structures, which is concomitant to virus production.

It is expected from these findings that a part of the punctae in which core accumulated represented assembly sites. To characterize the connection of these dots with replication sites, we investigated the intracellular localization of core punctae colocalizing with the non-structural proteins revealed here, that is, NS5A and NS4B (Figures 1 and 2). Interestingly, both structures frequently localized (approximately $40 \%-55 \%$ ) at the edge of LDs in Jc1infected cells (Figure $5 D$ ). By super-resolution microscopy, we found that core/NS4B dotted structures apposed at LD edges exhibited a bipolar arrangement, separating by approximately $0.1 \mu \mathrm{m}$ core on the one side and NS4B on the other side (Figure $3 B$ ). Importantly, a similarly high proportion of core/NS4B and core/NS5A structures remained apposed at the edges of LDs in infected cells treated or not

\section{B}
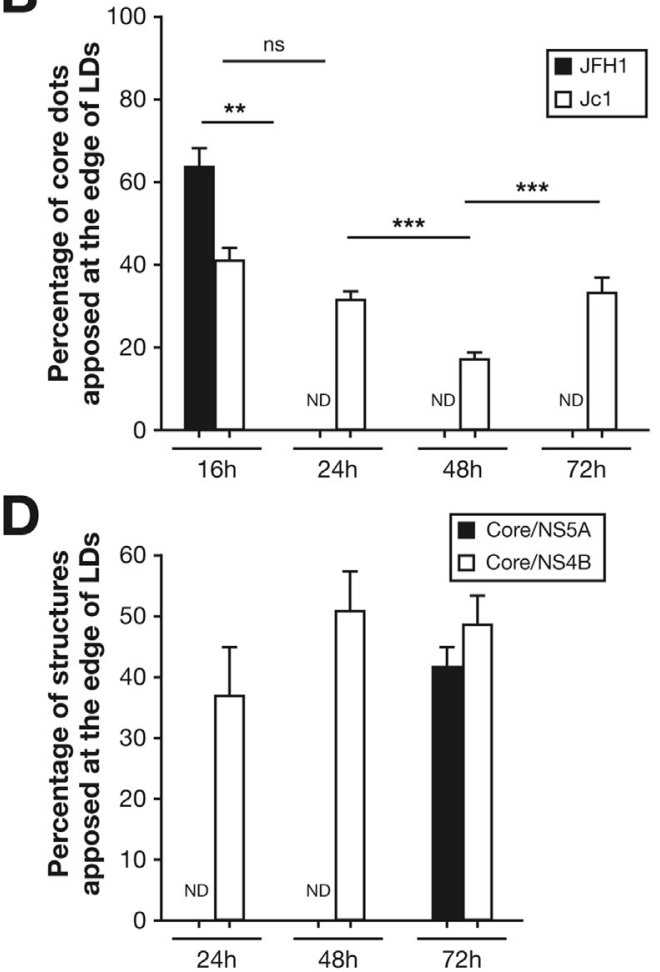

Figure 5. Characterization of HCV core structures within infected cells. A schematic representation distinguishing the different categories of core structures with regard to LD localization that were quantified $(A)$. From images of Supplementary Figure $8 A$, the percentage of core punctate structures apposed at the edge of LDs $(B)$ and the percentage of LDs with associated core either on ER membrane or on LD surface $(C)$ were quantified using a macro developed with ImageJ (see Supplementary Results). The percentage of core/NS4B and core/NS5A punctate structures apposed at the edge of LDs in Jc1-infected cells $(D)$ were quantified with ImageJ. ND, not determined. For each condition, $30-50$ cells were quantified. ${ }^{\star \star \star} P \leq .001$; ${ }^{\star \star} P \leq .01$; ns (not significant) $P>.05$. 


with DCV (Supplementary Figure 6B), which further implied that DCV did not inhibit HCV assembly by altering localization of assembly proteins.

\section{Daclatasvir Prevents Hepatitis C Virus Genome Transfer to Virus-Induced Structures That May Represent Assembly Platforms}

At first sight, the increasing size of E2/core/NS4B structures during the infection course (Figure 4) seemingly contradicted the fact that DCV treatment could also induce their enlargement (Figure 2). As DCV inhibits viral particle assembly, we thought that this apparent paradox reflects the accumulation of proteins at assembly and RC sites. To test this hypothesis, we reasoned that impairing cellular assembly factors would similarly induce the expansion of E2/core/NS5A and/or E2/core/NS4B structures. Hence, we targeted diacylglycerol acyltransferase- 1 , a factor that regulates HCV assembly by attracting core and NS5A proteins onto the surface of the same LDs. ${ }^{26,27}$ Upon diacylglycerol acyltransferase-1 down-regulation or chemical inhibition, which reduced infectivity by approximately 9 -fold, we found that both E2/core/NS5A and/or E2/core/NS4B structures enlarged, by up to 2-fold (Supplementary Figure 9). In a similar manner, we surmised that, owing to the dual role of NS5A in replication vs packaging of HCV RNA, ${ }^{8,18}$ the enlargement of either structure upon short-time DCV treatment (Figures 1 and 2) may reflect a loss of functional transition from HCV replication to assembly as caused by defective NS5A targeting of the viral genome to assembly components, ultimately resulting in their aggregation as inactive structures.

To further address this possibility, we wanted to investigate whether the viral genome and its replicative intermediate, that is, HCV $\mathrm{RNA}(+)$ and $\mathrm{RNA}(-)$ species, respectively, could be found within these structures and could be altered upon DCV treatment. We therefore combined immunofluorescence analysis to label core, NS4B, and NS5A proteins with fluorescence in situ hybridization to detect HCV RNA(+) and RNA(-) strands, which appeared as dots that could represent sites of translation, replication, viral assembly, or intracellular particles. ${ }^{28}$ Importantly, both $\mathrm{HCV}$ RNA(+) and RNA(-) were detected within core/NS5A and core/NS4B structures, which suggested that they represent, at least partly, assembly structures connected with RCs (Figure $6 A-C$, panels $i$ and ii). Interestingly, a 6-hour-long DCV treatment did not change the proportion of either protein structure colocalizing with both types of RNAs (Figure 6A-C, panels $i$ and ii). However, when we quantified the number of RNA dots colocalizing with HCV protein dots in Jc1-infected cells, we found that such DCV treatment specifically decreased by up to 3 -fold the proportion of HCV RNA(+) dots colocalizing with either core or NS4B punctae, whereas no changes were observed in Jc1Y93H infected cells (Figure $6 A$ and $B$, panels iii and $i v$ ). In contrast, the proportion of $\mathrm{HCV} \operatorname{RNA}(+)$ dots colocalizing with NS5A structures remained unchanged (Figure $6 \mathrm{~A}$ and $B$, panel $v$ ), implying that DCV-mediated NS5A inhibition acts downstream of NS5A/RNA interaction(s), in agreement with previous reports indicating that DCV does not inhibit HCV RNA binding to NS5A. ${ }^{14,29}$ Finally, DCV did not change the proportion of $\mathrm{HCV} \operatorname{RNA}(-)$ colocalizing with NS4B, NS5A, and core in either Jc1 or Jc1-Y93H-infected cells (Figure $6 \mathrm{~A}$ and $C$ ). Together these observations suggest that DCV inhibited assembly by blocking the formation of active, that is, $\mathrm{RNA}(+)$ containing, assembly sites.

\section{Discussion}

Our results uncover novel features of DCV-mediated HCV inhibition that further our understanding of HCV assembly. We confirmed that DCV induces NS5A clustering, in line with previous studies. ${ }^{5,12,15}$ Interestingly, we found that other HCV proteins, such as E2, core, and NS4B, gathered with these NS5A clusters (Figures 1 and 2, Supplementary Figure 4). We also found that the enlargement of these clusters upon short-time DCV treatments correlated with the reduction of viral particle assembly. As we will discuss, we propose that DCV-induced accretion of HCV proteins reflects the loss of functional association between HCV replication and assembly.

\section{Connection Between Replication Complexes and Assembly Sites at Lipid Droplets Proximity}

Overall, our imaging, biochemical, and functional data are congruent with a model previously proposed by others ${ }^{21}$ wherein assembly sites are closely associated with RCs and LDs in a micro-environment favoring replication, mobilization, and protection of HCV RNA. First, by comparing the poorly infectious JFH1 virus vs the highly infectious Jc1 virus, we found that the occurrence and intracellular localization of E2/core/NS4B and E2/core/ NS5A structures were intimately related to infectivity levels (Figure 4) and that such structures were frequently apposed at the edge of LDs, that is, close to the ER membrane, but were not associated around or at LD surface (Figure 5). Second, through time-course analysis of infection combined with translation inhibition assays (Supplementary Figure 8), we highlighted the progressive

Figure 6. DCV prevents the delivery of $\mathrm{HCV} \mathrm{RNA}(+)$ to assembly sites. Cells infected with Jc1 or Jc1-Y93H viruses $(\mathrm{MOI}=0.2)$ were incubated at 66 hours post-infection with $1 \mathrm{nM}$ DCV or dimethyl sulfoxide (DMSO) for 6 hours. After staining for HCV core, NS4B, and NS5A proteins and nuclei, HCV RNA(+) and RNA(-) species were stained by fluorescent in situ hybridization. Colocalization of core (red channel) with NS4B or NS5A proteins (green channel) and HCV RNA $(+)$ or RNA $(-)$ strands (blue channel) was analyzed by confocal microscopy $(A)$. Nuclei are represented as cyan channel. Scale bars of panels and zooms from squared area represent $10 \mu \mathrm{m}$ and $2 \mu \mathrm{m}$, respectively. The proportion of core/NS4B and core/NS5A colocalizing with HCV RNA $(+)(B)$ or RNA $(-)(C)$, as well as the proportion of HCV RNA $(+)(B)$ or RNA $(-)(C)$ dots colocalizing with core, NS4B, or NS5A structures were quantified with ImageJ. For each condition, $30-50$ cells were quantified. ${ }^{\star \star \star \star} P \leq .0001 ;{ }^{\star \star \star} P \leq .001$; ${ }^{\star \star} P \leq .01 ;{ }^{*} P \leq .05$; ns (not significant) $P>.05$. 
accumulation of core punctae at ER membranes, which ultimately appeared as a mesh distributed throughout the ER, and their rapid and strong colocalization with HCV glycoproteins. Third, we found that a significant proportion (approximately 15\%-20\%) of core/NS4B and core/NS5A structures contain HCV RNAs (Figure 6). Finally, our data underscored that the structures associating $\mathrm{RNA}(+)$, core, and E2 structural components (ie, representing, at least in part, assembly sites ${ }^{28}$ ) with RCs and LDs, ${ }^{21}$ evolve in a very dynamic process both naturally and in response to specific inhibitors.

\section{Daclatasvir Induces Clustering of Viral Proteins by Altering Both Assembly and Replication Complex Formation}

We noticed that a high proportion (approximately 80\%85\%) of core/NS4B and core/NS5A structures do not contain HCV RNAs. We propose that such structures may represent assembly sites for which functional linkage with RCs or, alternatively, viral RNA transfer to nascent virions, have waned or not occurred. Intuitively, one might expect that functional assembly sites would be difficult to seize, as they are necessarily transient, owing to release of assembled viral particles from such sites into the ER lumen and secretion by the cell. In addition, that HCV RNA seems ratelimiting for inducing functional assembly is congruent with other findings that functional RCs, represented by DMVs having a cytosol-oriented orifice allowing release of newly synthesized HCV RNA, account for $<10 \%,{ }^{10}$ and that HCV RNA triggers viral particle envelopment. ${ }^{18}$ Furthermore, $\mathrm{HCV}$ assembly is a poorly efficient process, in line with a general notion that the vast majority of structural proteins are not engaged or consummated in virions production. Accordingly, the progressive accumulation and enlargement of core-dotted structures appearing as a mesh in Jc1infected cells (Supplementary Figure 8) likely revealed accretion of nonfunctional assembly sites or, corollary, of residual core proteins that have not engaged in assembled and/or released virions. In support of this view, we found that such core punctae do not arise from mobilization of LDassociated core, but rather from their multiplication throughout the ER (Supplementary Figure 8) while replication and translation progress, and from their enlargement after ER targeting.

In this respect, our unexpected finding that DCV accentuated the clustering and/or enlargement of core punctae concomitant to inhibition of assembly may likely reflect an accrued accumulation of nonfunctional assembly sites, that is, of core proteins not recruited or released as infectious viral particles. Furthermore, we observed that both E2/ core/NS4B and E2/core/NS5A structures also enlarged upon DCV treatment, concomitant to assembly inhibition. Finally, inhibiting assembly by targeting diacylglycerol acyltransferase-1, which promotes localization of core and NS5A at LDs, ${ }^{26,27}$ also resulted in enlargement of either structure (Supplementary Figure 9). However, these observations are not paradoxical if one considers that a majority of such structures are not functionally connected to ongoing assembly events. Indeed, expression of HCV proteins continuously raises several vesicle types that are formed from the ER membrane, particularly viral particles and DMVs. Therefore, the release of the 2 latter vesicles, respectively, within the ER lumen and the cytosol, as well as their turnover through secretion and/or degradation, requires an unceasing creation of areas forming novel assembly sites and RCs. In this regard, NS5A, which intersects cellular degradation machineries, for example, autophagy, ${ }^{30}$ might be pivotal for DMV turnover.

Importantly, because the Y93H mutation in NS5A renders both assembly and DMV biogenesis events resistant to DCV, ${ }^{6,9}$ it implies that DCV targets a mutual, specific NS5A function that inhibits both processes. Indeed, NS5A, in concert with $\mathrm{CypA}^{9,31}$ and NS4B, ${ }^{10,11,32,33}$ has the capacity to induce DMV biogenesis through binding of its $\mathrm{AH}$ N-terminal amphipathic helix on the ER membrane and, as proposed previously, ${ }^{1,6,34-36}$ NS5A multimerization. Because DCV binds close to the amino-terminus of the DI domain of NS5A dimer, that is, at the junction of both DI subunits and their interface with the ER membrane, ${ }^{1,6,7,35}$ DCV binding could perturb the positioning, orientation, or flexibility of the $\mathrm{AH}$ helix that anchors NS5A. This could alter the membranotropic properties of NS5A by altering its membrane binding, multimerization, and/or interaction with partners, which would explain DCV capacity to inhibit the formation of DMVs $^{6,9}$ and subsequent RNA synthesis and/or trafficking, but also its lack of effect on replication within pre-existing DMVs. ${ }^{9}$ Owing to the fast turnover of DMVs, ${ }^{6}$ this inhibition would progressively lead to decay of HCV RNAs ${ }^{5}$ by loss of newly formed DMVs. As a result, de novo-expressed nonstructural proteins would tend to accumulate because their lack of segregation as DMVs would enhance the clustering of

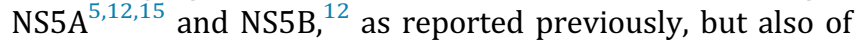
NS4B (this report) at ER membrane domains where DMVs form.

\section{Daclatasvir Prevents Delivery of Viral Genomes to Assembly Sites}

It is unlikely that inhibition of DMV biogenesis explains the nearly immediate effect of DCV on assembly of infectious particles. ${ }^{4-6}$ However, another important finding of our study was that short-time DCV treatments that prevented HCV assembly strongly reduced the colocalization of core with $\mathrm{HCV} \mathrm{RNA}(+)$ strand (Figure 6), which likely precluded formation of infectious particles (Supplementary Figure 1). Furthermore, because DCV had no effect on colocalization of viral RNA with core/NS4B and core/NS5A structures (Figure 6), we propose that, reminiscent of specific NS5A mutants in domain DIII that affect core/NS5A interaction, ${ }^{18}$ this could be due to a lack of transfer of viral genomic RNA to assembling virions. Indeed, DCV-mediated inhibition of DMV formation ${ }^{6,9}$ may not result, per se, in the blockage of HCV RNA packaging or transfer because RNA synthesis may not occur before DMVs become fully formed and active. ${ }^{5}$ Rather, we propose that DCV restrains preexisting, replication-active DMVs, that is, among those already present at the onset of DCV treatment, from 
mediating this critical event in infectious particles assembly by targeting the same function of NS5A as described here, that is, its membranotropic properties. Indeed, transfer of HCV RNA from active DMVs to assembly sites would also be precluded if the membrane-binding properties of NS5A were altered for different, albeit not exclusive reasons. First, as NS5A is located both inside and outside DMVs, ${ }^{37}$ such alterations would compromise the stability of DMV surface-associated NS5A, and, consequently, the gathering of replication-active DMVs at the vicinity of HCV particle assembly sites and/or of LDs, because this targeting depends on core and NS5A interaction $^{21,38,39}$ and is critical for mediating RNA transfer to the core protein. ${ }^{18}$ Second, as NS5A is also distributed on LDs and ER membranes, ${ }^{10,21,27}$ DCV-mediated disruption of NS5A membrane association on these interfaces may subsequently inhibit RNA transfer, which could require NS5A multimers to guide HCV RNA toward structural proteins, as proposed elsewhere. ${ }^{1,6,34-36}$ Regardless of NS5A localization, this would explain why DCV did not alter RNA/NS5A colocalization (Figure 6), as DCV does not inhibit RNA/ NS5A binding, ${ }^{14,29}$ whereas perturbing NS5A multimers by DCV via either their disruption or altered membrane association would likely have a strong impact on RNA transfer and packaging in assembling virions. Third, DCVinduced perturbation of NS5A amino-terminus, which interacts with NS4B C-terminal domain, ${ }^{40,41}$ may preclude NS4B packaging functions ${ }^{42}$ and RNA transfer to core. In this respect, it is interesting to note that DCV treatment also strongly reduced the colocalization of NS4B and HCV $\mathrm{RNA}(+)$ strand (Figure 6). This likely reflects the role of NS4B in post-replication steps, ${ }^{32,43}$ particularly the packaging function of its C-terminal domain, ${ }^{42}$ which relies on both weak RNA binding capacity ${ }^{44}$ and interaction with the DIII domain of NS5A that is involved in $\mathrm{HCV}$ assembly. $^{18,38,39,45}$

Interestingly, enlarged E2/core/NS5A and E2/core/ NS4B structures were observed for the Y93H DCV-resistant virus as compared to wild-type in absence of DCV (Figures 1 and 2). Nonetheless, RNA(+) dots readily localized with core or NS4B structures (Figure 6B), even in the presence of DCV (Supplementary Figure 1), congruent with efficient viral particle assembly of this mutant. Therefore, in line with the model discussed here, we propose that DCV-escape mutations may compensate altered membranotropic properties of NS5A and optimize interactions with NS4B (and/or other nonstructural proteins) in order to maintain efficient RNA targeting to assembly sites. Additional experiments will be required to validate this hypothesis.

In conclusion, our results indicate that DCV may inhibit HCV by preventing NS5A-mediated HCV RNA transfer to sites of virion assembly, in addition to other effects induced by this multifunctional protein. ${ }^{8}$ This finding emphasizes the attractiveness of this class of inhibitors that targets 2 distinct steps of HCV cycle, that is, RNA replication and viral particle assembly. Furthermore, investigating the mode of action of DCV allowed further characterization of how replication and assembly are intimately linked and a specific function of NS5A in this respect.

\section{Supplementary Material}

Note: To access the supplementary material accompanying this article, visit the online version of Gastroenterology at www.gastrojournal.org, and at http://dx.doi.org/10.1053/ j.gastro.2016.11.047.

\section{References}

1. Bartenschlager R, Lohmann V, Penin F. The molecular and structural basis of advanced antiviral therapy for hepatitis C virus infection. Nat Rev Microbiol 2013; 11:482-496.

2. Gotte M, Feld JJ. Direct-acting antiviral agents for hepatitis C: structural and mechanistic insights. Nat Rev Gastroenterol Hepatol 2016;13:338-351.

3. Gao M, Nettles RE, Belema M, et al. Chemical genetics strategy identifies an HCV NS5A inhibitor with a potent clinical effect. Nature 2010;465:96-100.

4. Guedj J, Dahari H, Rong L, et al. Modeling shows that the NS5A inhibitor daclatasvir has two modes of action and yields a shorter estimate of the hepatitis $\mathrm{C}$ virus half-life. Proc Natl Acad Sci U S A 2013;110:3991-3996.

5. McGivern DR, Masaki T, Williford S, et al. Kinetic analyses reveal potent and early blockade of hepatitis $\mathrm{C}$ virus assembly by NS5A inhibitors. Gastroenterology 2014;147:453-462 e7.

6. Berger C, Romero-Brey I, Radujkovic D, et al. Daclatasvir-like inhibitors of NS5A block early biogenesis of hepatitis $C$ virus-induced membranous replication factories, independent of RNA replication. Gastroenterology 2014;147:1094-1105 e25.

7. Ascher DB, Wielens J, Nero TL, et al. Potent hepatitis C inhibitors bind directly to NS5A and reduce its affinity for RNA. Sci Rep 2014;4:4765.

8. Ross-Thriepland D, Harris M. Hepatitis C virus NS5A: enigmatic but still promiscuous 10 years on. J Gen Virol 2015;96:727-738.

9. Chatterji U, Bobardt M, Tai A, et al. Cyclophilin and NS5A inhibitors, but not other anti-hepatitis C virus (HCV) agents, preclude HCV-mediated formation of doublemembrane-vesicle viral factories. Antimicrob Agents Chemother 2015;59:2496-2507.

10. Romero-Brey I, Merz A, Chiramel A, et al. Threedimensional architecture and biogenesis of membrane structures associated with hepatitis C virus replication. PLoS Pathog 2012;8:e1003056.

11. Romero-Brey I, Berger C, Kallis S, et al. NS5A Domain 1 and Polyprotein cleavage kinetics are critical for induction of double-membrane vesicles associated with hepatitis C Virus replication. MBio 2015;6:e00759.

12. Chukkapalli V, Berger KL, Kelly SM, et al. Daclatasvir inhibits hepatitis C virus NS5A motility and hyperaccumulation of phosphoinositides. Virology 2015; 476:168-179.

13. Lee $\mathrm{C}, \mathrm{Ma} H$, Hang JQ, et al. The hepatitis C virus NS5A inhibitor (BMS-790052) alters the subcellular localization of the NS5A non-structural viral protein. Virology 2011; 414:10-18.

14. Targett-Adams P, Graham EJ, Middleton J, et al. Small molecules targeting hepatitis C virus-encoded NS5A 
cause subcellular redistribution of their target: insights into compound modes of action. J Virol 2011; 85:6353-6368.

15. Reghellin V, Donnici L, Fenu S, et al. NS5A inhibitors impair NS5A-phosphatidylinositol 4-kinase IIlalpha complex formation and cause a decrease of phosphatidylinositol 4-phosphate and cholesterol levels in hepatitis C virus-associated membranes. Antimicrob Agents Chemother 2014;58:7128-7140.

16. Huang L, Hwang J, Sharma SD, et al. Hepatitis C virus nonstructural protein 5A (NS5A) is an RNA-binding protein. J Biol Chem 2005;280:36417-36428.

17. Foster TL, Belyaeva T, Stonehouse NJ, et al. All three domains of the hepatitis C virus nonstructural NS5A protein contribute to RNA binding. J Virol 2010; 84:9267-9277.

18. Zayas M, Long G, Madan V, et al. Coordination of hepatitis $\mathrm{C}$ virus assembly by distinct regulatory regions in nonstructural protein 5A. PLoS Pathog 2016; 12:e1005376.

19. Pietschmann T, Kaul A, Koutsoudakis G, et al. Construction and characterization of infectious intragenotypic and intergenotypic hepatitis $\mathrm{C}$ virus chimeras. Proc Natl Acad Sci U S A 2006;103:7408-7413.

20. Boson B, Granio O, Bartenschlager R, et al. A concerted action of hepatitis $\mathrm{C}$ virus $\mathrm{p} 7$ and nonstructural protein 2 regulates core localization at the endoplasmic reticulum and virus assembly. PLoS Pathog 2011;7:e1002144.

21. Miyanari Y, Atsuzawa K, Usuda N, et al. The lipid droplet is an important organelle for hepatitis $\mathrm{C}$ virus production. Nat Cell Biol 2007;9:1089-1097.

22. Qiu D, Lemm JA, O'Boyle DR 2nd, et al. The effects of NS5A inhibitors on NS5A phosphorylation, polyprotein processing and localization. J Gen Virol 2011; 92:2502-2511.

23. Fridell RA, Qiu D, Valera L, et al. Distinct functions of NS5A in hepatitis C virus RNA replication uncovered by studies with the NS5A inhibitor BMS-790052. J Virol 2011;85:7312-7320.

24. Shavinskaya A, Boulant S, Penin F, et al. The lipid droplet binding domain of hepatitis $\mathrm{C}$ virus core protein is a major determinant for efficient virus assembly. J Biol Chem 2007;282:37158-37169.

25. Boulant S, Targett-Adams P, McLauchlan J. Disrupting the association of hepatitis $\mathrm{C}$ virus core protein with lipid droplets correlates with a loss in production of infectious virus. J Gen Virol 2007;88:2204-2213.

26. Herker E, Harris C, Hernandez C, et al. Efficient hepatitis C virus particle formation requires diacylglycerol acyltransferase-1. Nat Med 2010;16:1295-1298.

27. Camus G, Herker E, Modi AA, et al. Diacylglycerol acyltransferase- 1 localizes hepatitis $C$ virus NS5A protein to lipid droplets and enhances NS5A interaction with the viral capsid core. J Biol Chem 2013;288:9915-9923.

28. Shulla A, Randall G. Spatiotemporal analysis of hepatitis C virus infection. PLoS Pathog 2015;11:e1004758.

29. Nag A, Robotham JM, Tang H. Suppression of viral RNA binding and the assembly of infectious hepatitis $\mathrm{C}$ virus particles in vitro by cyclophilin inhibitors. J Virol 2012; 86:12616-12624.
30. Kim N, Kim MJ, Sung PS, et al. Interferon-inducible protein SCOTIN interferes with HCV replication through the autolysosomal degradation of NS5A. Nat Commun 2016;7:10631.

31. Madan V, Paul D, Lohmann V, et al. Inhibition of HCV replication by cyclophilin antagonists is linked to replication fitness and occurs by inhibition of membranous web formation. Gastroenterology 2014;146:1361-1372 e1-e9.

32. Paul D, Romero-Brey I, Gouttenoire J, et al. NS4B selfinteraction through conserved $\mathrm{C}$-terminal elements is required for the establishment of functional hepatitis $\mathrm{C}$ virus replication complexes. J Virol 2011;85:6963-6976.

33. Gouttenoire J, Montserret R, Paul D, et al. Aminoterminal amphipathic alpha-helix $\mathrm{AH} 1$ of hepatitis $\mathrm{C}$ virus nonstructural protein 4B possesses a dual role in RNA replication and virus production. PLoS Pathog 2014; 10:e1004501.

34. Appel N, Schaller T, Penin F, et al. From structure to function: new insights into hepatitis C virus RNA replication. J Biol Chem 2006;281:9833-9836.

35. Lambert SM, Langley DR, Garnett JA, et al. The crystal structure of NS5A domain 1 from genotype 1a reveals new clues to the mechanism of action for dimeric HCV inhibitors. Protein Sci 2014;23:723-734.

36. Love RA, Brodsky O, Hickey MJ, et al. Crystal structure of a novel dimeric form of NS5A domain I protein from hepatitis C virus. J Virol 2009;83:4395-4403.

37. Paul D, Hoppe S, Saher G, et al. Morphological and biochemical characterization of the membranous hepatitis C virus replication compartment. J Virol 2013; 87:10612-10627.

38. Masaki T, Suzuki R, Murakami K, et al. Interaction of hepatitis C virus nonstructural protein 5A with core protein is critical for the production of infectious virus particles. J Virol 2008;82:7964-7976.

39. Appel N, Zayas M, Miller S, et al. Essential role of domain III of nonstructural protein 5A for hepatitis C virus infectious particle assembly. PLoS Pathog 2008;4:e1000035.

40. Biswas A, Treadaway J, Tellinghuisen TL. Interaction between non-structural proteins NS4B And NS5A is essential for proper NS5A localization and hepatitis C virus RNA replication. J Virol 2016;90:7205-7218.

41. David N, Yaffe Y, Hagoel L, et al. The interaction between the hepatitis C proteins NS4B and NS5A is involved in viral replication. Virology 2015;475:139-149.

42. Han Q, Manna D, Belton K, et al. Modulation of hepatitis $\mathrm{C}$ virus genome encapsidation by nonstructural protein 4B. J Virol 2013;87:7409-7422.

43. Jones DM, Patel AH, Targett-Adams $P$, et al. The hepatitis C virus NS4B protein can trans-complement viral RNA replication and modulates production of infectious virus. J Virol 2009;83:2163-2177.

44. Einav S, Gerber D, Bryson PD, et al. Discovery of a hepatitis $\mathrm{C}$ target and its pharmacological inhibitors by microfluidic affinity analysis. Nat Biotechnol 2008; 26:1019-1027.

45. Tellinghuisen TL, Foss KL, Treadaway JC, et al. Identification of residues required for RNA replication in domains II and III of the hepatitis C virus NS5A protein. J Virol 2008;82:1073-1083. 
Author names in bold designate shared co-first authorship.

Received July 20, 2016. Accepted November 28, 2016.

Reprint requests

Address requests for reprints to: François-Loïc Cosset, PhD, International Center for Infectiology Research, Université de Lyon, Lyon, 21 Avenue Tony Garnier, Lyon 69365, France. e-mail: flcosset@ens-lyon.fr; fax: +33 47272 8137.

Acknowledgments

The authors are grateful to Ralf Bartenschlager for the gift of JFH1, Jc1, and Jc1-Y93H constructs, Charles Rice for the gift of Huh7.5 cells and 9E10 monoclonal NS5A antibody, Mishinori Kohara for the gift of NS4B antibody,
Jane McKeating for the gift of 3/11 E2 antibody, and Colette Jolivet for the gift of core antibody. The authors thank François Penin for helpful comments and critical reading of the manuscript and Claire Lionnet and Elodie Chatre for excellent technical assistance with confocal microscopy.

The authors dedicate this paper to the memory of our colleague and friend Professor Christophe Terzian.

Marlène Dreux and François-Loïc Cosset contributed equally to this work.

\section{Conflicts of interest}

The authors disclose no conflicts.

\section{Funding}

This work was supported by the Agence Nationale de la Recherche sur le Sida et les Hépatites Virales (ANRS), European Research Council (ERC-2008-AdG233130-HEPCENT), and LabEx Ecofect (ANR-11-LABX-0048). 


\section{Supplementary Materials and Methods}

\section{Antibodies and Reagents}

Rabbit antiserum against calnexin (Sigma-Aldrich, Lyon, France), mouse anti-adipose differentiation-related protein (clone AP125; Progen, Heidelberg, Germany), mouse anti-Actin (clone AC74; Sigma-Aldrich), rabbit anti- diacylglycerol acyltransferase-1 (DGAT1) (clone H-255; Santa Cruz Biotechnology, Santa Cruz, CA), mouse anti-core 19D9D6 (kind gift from C. Jolivet, bioMérieux, Lyon, France), rat anti-E2 clone 3/11 (kind gift from J. McKeating, University of Birmingham, UK), mouse anti-NS5A 9E10 (kind gift from C. Rice, Rockefeller University, New York, NY), rabbit antiNS4B (kind gift from M. Kohara, Tokyo Metropolitan Institute of Medical Science, Tokyo, Japan), DCV (BMS-790052; Selleck Chemicals, Houston, TX), A922500 (Sigma-Aldrich), CHX (Sigma-Aldrich), Mowiol 40-88 (Fluka), Bodipy 493/503 and Hoecsht 33342 (Molecular Probes Europe BV), Fluoromount-G (SouthernBiotech), Protease/phosphatase inhibitor cocktail (Cell Signaling Technology, Danvers, MA) was used according to manufacturer's instructions.

\section{Inhibition of Diacylglycerol Acyltransferase-1}

Down-regulation of DGAT1 was achieved using procedure described previously ${ }^{1}$ via a small interfering RNA (5'-GAACCTCATCAAGTATGGCAT) expressed from a lentiviral vector (MISSION pLKO.1-puro vector with shorthairpin RNA insert TRCN0000036153; Sigma-Aldrich). DGAT1 expression was verified by Western blot analysis. Inhibition of DGAT1 was achieved using $150 \mu \mathrm{M}$ A922500 inhibitor added at the time of infection for 72 hours.

\section{Analysis of Intracellular and Extracellular RNA Levels}

RNAs were isolated from cells and supernatant harvested in guanidinium thiocyanate citrate buffer (GTC), extracted by phenol/chloroform extraction, reverse transcribed (iScript cDNA synthesis kit; Bio-Rad, Hercules, CA), and quantified with the FastStart Universal SYBR Green Master kit (Roche Applied Science, Indianapolis, IN) on an Applied StepOne Real-Time PCR apparatus, as described previously. ${ }^{2,3}$ The efficiency of extracellular RNA extraction and reverse transcription real-time quantitative polymerase chain reaction was controlled by the addition of carrier RNAs encoding in vitro transcripts of Xef1a (Xenopus transcription factor 1a) in supernatants diluted in GTC buffer. The sequences of the primers used for the reverse transcription real-time quantitative polymerase chain reaction were: HCV-specific primers (5'-CTGCGGAACCGGTGAGTA and 5'-TCAGGCAGTACCACAAGGC), glyceraldehyde-3-phosphate dehydrogenase-specific primers (5'-AGGTGAAGGTCGGAGTCAACG and $5^{\prime}$-TGGAAGATGGTGATGGGATTTC), and Xef1a-specific primers (5'-GACGTTGTCAC CGGGCACG and 5'-ACCAGGCATGGTGGTTACCTT TGC). Extracellular and intracellular HCV RNA levels were normalized for Xef1a and glyceraldehyde-3-phosphate dehydrogenase RNA levels, respectively.

\section{Analysis of Intracellular and Extracellular Core Protein Levels}

Infected cells were washed with PBS, trypsinized, and centrifuged for 5 minutes at $400 \times g$. Cell pellets were resuspended in PBS and subjected to 4 cycles of freeze and thaw using liquid nitrogen. Samples were then centrifuged at $10,000 \times g$ for 10 minutes to remove cell debris. For the quantification of extracellular core protein levels, supernatants of infected cells were harvested and diluted in Dulbecco's modified minimal essential medium to obtain a final concentration of $5 \%$ bovine serum albumin. Core protein levels were then quantified with the HCV core Antigen Detection ELISA assay (XpressBio, Frederick, MD) according to manufacturer's instructions.

\section{Subcellular Fractionation}

Separation of different membrane compartments was achieved as described previously. ${ }^{4}$ Huh7.5 cells were electroporated with in vitro-transcribed Jc1 RNA. Sixty-six hours post-electroporation, cells were washed 3 times and were incubated with $1 \mathrm{nM}$ DCV or dimethyl sulfoxide for an additional 6 hours. Cells were then detached, washed in PBS, and homogenized in 1 volume of $10 \mathrm{mM}$ HEPES-NaOH $10 \mathrm{mM}$ (pH 7.8) (hypo-osmotic buffer). Cells were allowed to swell on ice for 20 minutes and were re-isolated by centrifugation at $800 \times g$ at $4^{\circ} \mathrm{C}$ for 5 minutes. The medium was returned to iso-osmoticity by removing two-thirds of the supernatant and adding one-third volume of $0.6 \mathrm{M}$ sucrose and $10 \mathrm{mM}$ HEPES-NaOH at $\mathrm{pH} 7.8$ (hyper-osmotic buffer). Cells were disrupted by passaging 20 times through a 25-G needle and lysates were extracted from the nuclei by centrifugation at $13,000 \times g$ for 10 minutes at $4^{\circ} \mathrm{C}$. Subcellular fractionation was performed in 3-step iodixanol gradients. Equal protein amounts of the post-nuclear extracts were mixed with $60 \%$ iodixanol to give a final concentration of $30 \%$, the hypo-osmotic buffer was mixed with $60 \%$ iodixanol to generate $10 \%$ and $20 \%$ iodixanol solutions. Equal volumes of these 3 solutions were layered in SW60Ti centrifuge tubes and centrifuged at 50,000 rpm for 3 hours at $4{ }^{\circ} \mathrm{C}$. Twelve fractions were collected from the top and proteins were revealed by Western blotting.

\section{Western Blotting}

Infected cells were lysed in lysis buffer $(20 \mathrm{mM}$ Tris [pH 7.5], $1 \%$ Triton X100, $0.05 \%$ sodium dodecyl sulfate, 150 nM NaCL, 5\% $\mathrm{Na}$ deoxycholate) supplemented with protease/phosphatase inhibitor cocktail (Cell Signaling Technology) and clarified from the nuclei by centrifugation at $13,000 \times g$ for 10 minutes at $4^{\circ} \mathrm{C}$. Ten micrograms total proteins were separated by sodium dodecyl sulfate polyacrylamide gel electrophoresis, then transferred to polyvinylidene fluoride membranes (FluoroTrans W; Pall Corporation, Port Washington, NY) and revealed with specific monoclonal antibodies, followed by the addition of goat anti-mouse, anti-rat, or anti-rabbit immunoglobulin conjugated to peroxidase (Dako A/S, Glostrup, Denmark). The proteins of interest were revealed by enhanced chemiluminescence detection (SuperSignal West Pico 
Chemiluminescent, Thermo Scientific, Logan, UT) as recommended by the manufacturer.

\section{Supplementary Results}

For quantification of structures apposed at the edge of LDs, ${ }^{5}$ we developed an ImageJ macro that searches for variation of max intensity in the neighborhood of each LD as an indicator of proximity. The code of this macro is as can be found on pages e3-e5

\section{Supplementary References}

1. Lavillette D, Tarr AW, Voisset C, et al. Characterization of host-range and cell entry properties of hepatitis $\mathrm{C}$ virus of major genotypes and subtypes. Hepatology 2005; 41:265-274.
2. Calattini S, Fusil F, Mancip J, et al. Functional and biochemical characterization of hepatitis $\mathrm{C}$ virus $(\mathrm{HCV})$ particles produced in a humanized liver mouse model. J Biol Chem 2015;290:23173-23187.

3. Dao Thi VL, Granier C, Zeisel MB, et al. Characterization of hepatitis $\mathrm{C}$ virus particle subpopulations reveals multiple usage of the scavenger receptor BI for entry steps. J Biol Chem 2012;287:31242-31257.

4. Boson B, Granio O, Bartenschlager R, et al. A concerted action of hepatitis $C$ virus $p 7$ and nonstructural protein 2 regulates core localization at the endoplasmic reticulum and virus assembly. PLoS Pathog 2011;7:e1002144.

5. Boulant S, Targett-Adams P, McLauchlan J. Disrupting the association of hepatitis $\mathrm{C}$ virus core protein with lipid droplets correlates with a loss in production of infectious virus. J Gen Virol 2007;88:2204-2213. 
showMessage("The system will now search for variation of $\backslash$ nmax intensity in the neighborhood of each vesicle $\backslash$ nas indicator of proximity $\backslash n \backslash n$ The integrated density should increase dramatically too.");

//Image management

if (nImages $==0)\{$ \}else \{ exit("No images are open");

getDimensions(width, height, channels, slices, frames);

if(channels>1) run("Split Channels");

titres=newArray(nImages);

//setBatchMode(true);

for $(\mathrm{i}=1 ; \mathrm{i}<=$ nImages; $\mathrm{i}++)\{$

selectImage(i);

\}

titres[i-1]=getTitle();

dir=getDirectory("image");

Dialog.create("Parameters");

Dialog.addNumber("Number of dilatations", 3);

Dialog.addNumber("Max. variation of max intensity", 50);

Dialog.addNumber("Max. variation of integrated intensity", 2000);

Dialog.addNumber("Min. area for analysis", 0.02);

Dialog.addChoice("To dilate", titres);

Dialog.addChoice("Target", titres);

Dialog.addCheckbox("Save ROIs ?", 0);

Dialog.show();

n=Dialog.getNumber();

maxIntVar=Dialog.getNumber();

maxDenVar=Dialog.getNumber();

minArea=Dialog.getNumber();

toDilate=Dialog.getChoice $($;

target=Dialog.getChoice();

saveROI=Dialog.getCheckbox(0;

//Preparation and configuration

run("Set Measurements...", "area min integrated redirect=None decimal=3");

roiManager("reset");

//Removing of channels useless for this analysis and separation of channels

$/{ }^{*}$ setSlice(2);

run("Delete Slice", "delete=channel");

run("Next Slice [>]");

run("Delete Slice", "delete=channel");

run("Split Channels");

*/ 
$/^{*}$ TODO add sequence of automatic selection of the cell

run("Next Slice [>]");

run("Gaussian Blur...", "sigma=20 slice");

setAutoThreshold("Percentile dark");

setAutoThreshold("Percentile dark");

run("Convert to Mask");

doWand(265, 252);

*/

//Automatic thresholding and separation

selectImage(toDilate);

//Possibility to place a ROI around the cell

waitForUser("Place ROI around the cell to avoid \nexternal counting");

//ROI counting

selectImage(toDilate);

run("Analyze Particles...", "size="+minArea+"-Infinity circularity=0.00-1.00

show=Nothing add");

//Selection of the image carrying the information

selectImage(target);

//selectWindow("C1-Jc1LDcoreNS4B4.lsm");

//Highlighting of the counted area

roiManager("Show All without labels");

//Saving of the ROI

if(saveROI) roiManager("Save", "');

//Scanning of the ROI

for $(\mathrm{i}=0 ; \mathrm{i}<$ roiManager("count");i++) \{

roiManager("Select", i);

//Measure of the dilations

$\mathrm{j}=1$;

while $(\mathrm{j}<=\mathrm{n})\{$

run("Measure");

run("Enlarge...", "enlarge=1 pixel");

$\mathrm{j}++$;

\}//end of ROI measurement

//Construction secondary tables

//Table of max

$\operatorname{maxs}=\operatorname{new} \operatorname{Array}(\mathrm{n})$;

for $(\mathrm{z}=0 ; \mathrm{z}<\mathrm{n} ; \mathrm{z}++)\{$

$\operatorname{maxs}[\mathrm{z}]=$ getResult("Max", z);

\}

/*Array.print(maxs); 
$\operatorname{print}(\operatorname{maxs}[4])$;

Array.reverse(maxs);

Array.print(maxs);

print(maxs[4]);

Array.getStatistics(maxs, min, max, mean, stdDev);

print(stdDev);

print(delta(maxs))

$* /$

//Table of integrated intensities

intDen=newArray(n);

for $(\mathrm{y}=0 ; \mathrm{y}<\mathrm{n} ; \mathrm{y}++)\{$ intDen[y]=getResult("RawIntDen", y);

\}

//Decision of the positive character or not

$\operatorname{if}(($ delta(maxs) $>$ maxIntVar $) \|($ delta(intDen) $>$ maxDenVar $)\}\{$ print("ROI "+i+1+"\tpositif");

\}else\{

print("ROI "+i+1+"\tnegatif");

\}

//if(deltaMax<threshold) the area of max intensity is close by //if(deltaRawIntDen>threshold) progress thus closeness

//deletion of $\mathrm{n}$ first raws of the table

IJ.deleteRows(0, n-1);

\}//end of the scanning of the ROI

function $\operatorname{delta}(\mathrm{a})\{$

val $=0$;

for $(\mathrm{i}=1 ; \mathrm{i}<$ a.length; $\mathrm{i}++)\{$

val=val+a[i] $-\mathrm{a}[\mathrm{i}-1]$;

\}

return val;

\} 


\section{A}

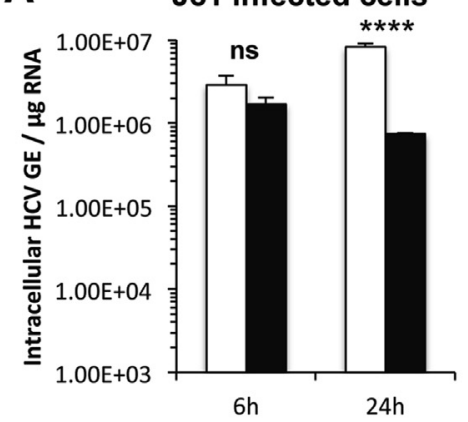

B

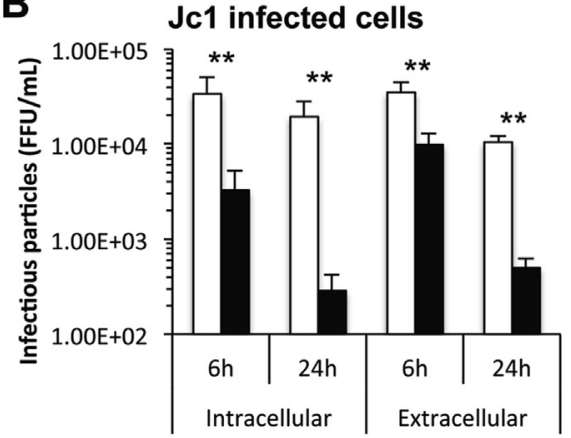

Jc1-Y93H infected cells
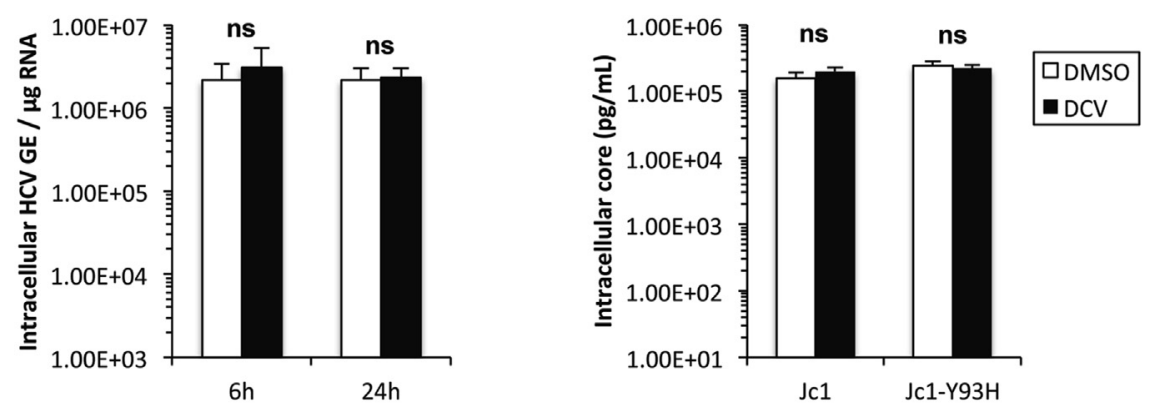

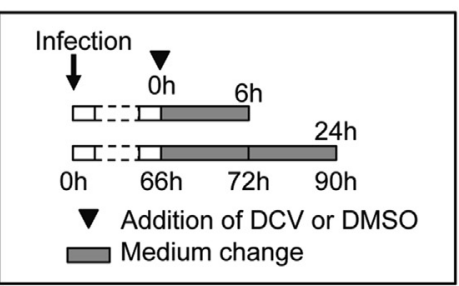

C
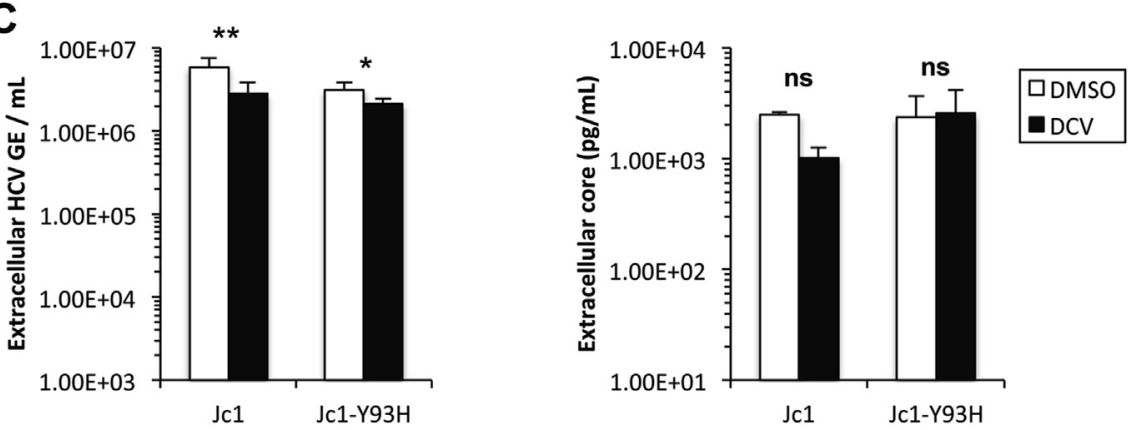

Supplementary Figure 1. DCV prevents HCV assembly. Huh7.5 cells infected with Jc1 or Jc1-Y93H viruses (MOI $=0.2)$ were incubated 66 hours post-infection with $1 \mathrm{nM}$ DCV or dimethyl sulfoxide (DMSO) for 6 hours vs 24 hours, as outlined in the boxed panel. (A) The intracellular viral genomes (left panels) and core proteins (right panel) were quantified by reverse transcription quantitative polymerase chain reaction (RT-qPCR) and enzyme-linked immunosorbent assay (ELISA), respectively. $(B)$ The intracellular or extracellular infectivity was determined from cell lysates or supernatants of infected cells, respectively. (C) The extracellular physical particles were analyzed by quantification of the release of viral RNA (left panel) or core protein (right pane/) in the supernatant by RT-qPCR and ELISA, respectively. GE, genome equivalent. ${ }^{\star \star \star} P \leq .0001 ;{ }^{\star \star \star} P \leq .001$; ${ }^{* *} P \leq .01 ;{ }^{*} P \leq .05 ;$ ns (not significant) $P>.05$. 
A

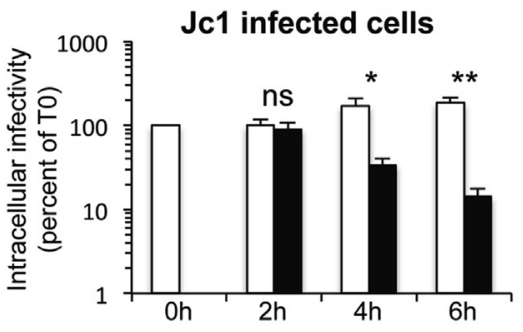

B

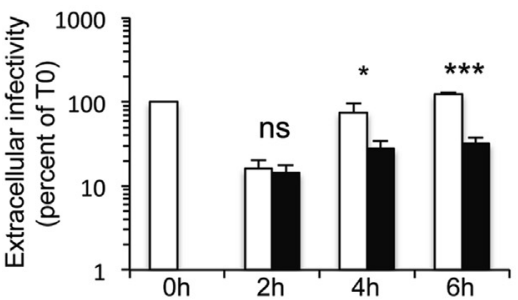

C

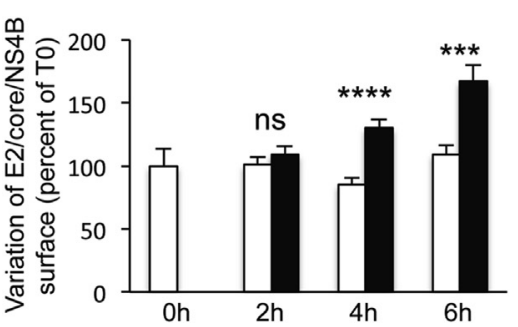

D

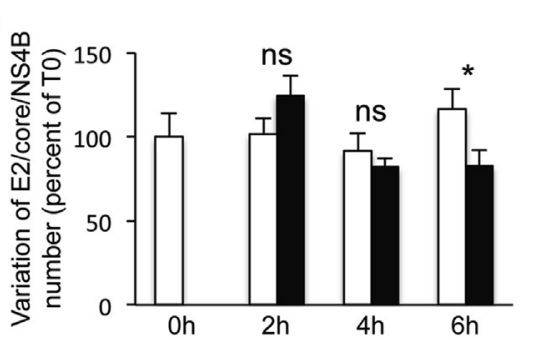

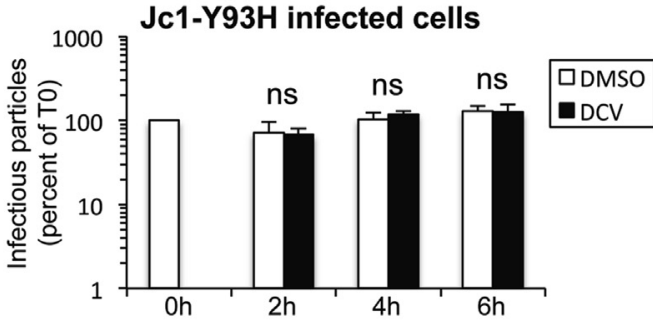
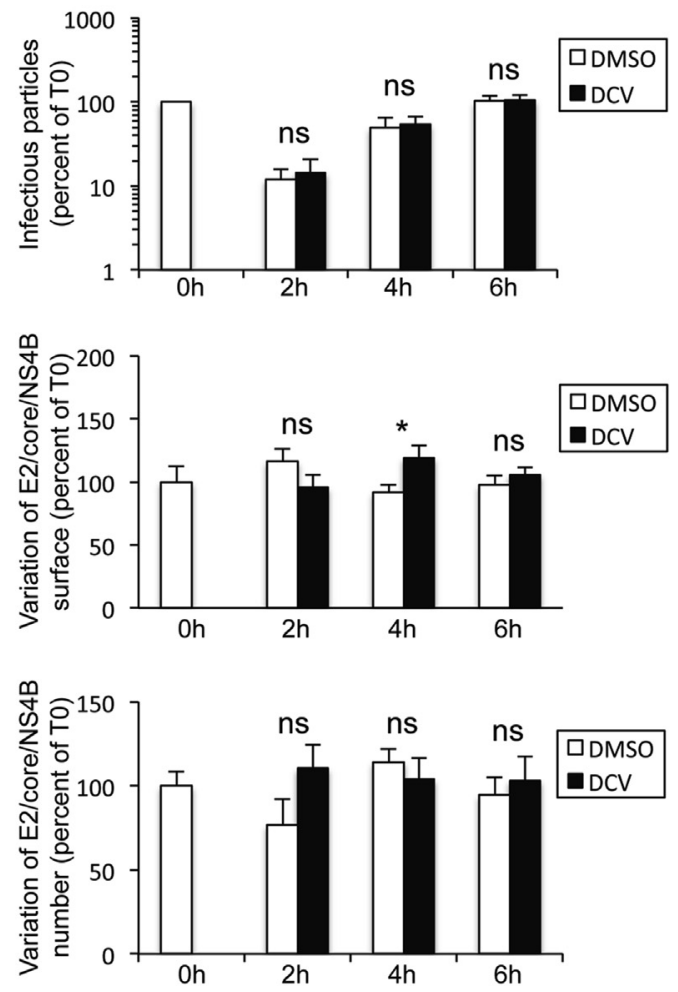

Supplementary Figure 2. HCV assembly inhibition by DCV correlates with clustering of structural proteins and NS4B. Huh7.5 cells were infected with $\mathrm{Jc} 1$ or $\mathrm{Jc} 1-\mathrm{Y} 93 \mathrm{H}$ viruses at an $\mathrm{MOI}$ of 0.2 . At 66 hours post-infection, cells were washed and incubated with $1 \mathrm{nM}$ of DCV or dimethyl sulfoxide (DMSO) for the indicated time, as outlined in the boxed panel. (A) The intracellular and $(B)$ extracellular infectivity were determined from cell lysates or supernatants of infected cells, respectively. $(C)$ The size and $(D)$ number of E2/core/NS4B structures were quantified with ImageJ. For each time point, results are expressed as percentage of the value at the initiation of DCV treatment (T0). For each condition, $30-50$ cells were quantified. ${ }^{\star \star \star \star} P \leq .0001 ;{ }^{\star \star \star} P \leq .001$; ${ }^{*} P \leq .01 ;{ }^{*} P \leq .05 ;$ ns (not significant) $P>.05$. 
A
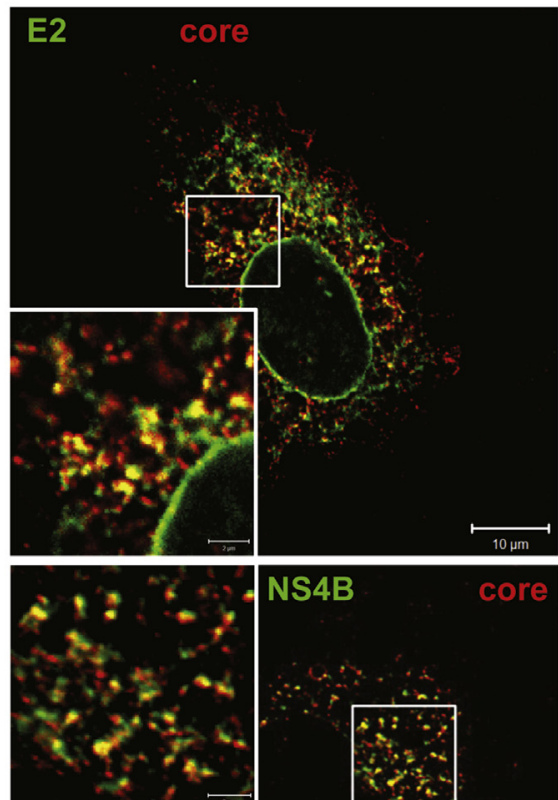

NS4B
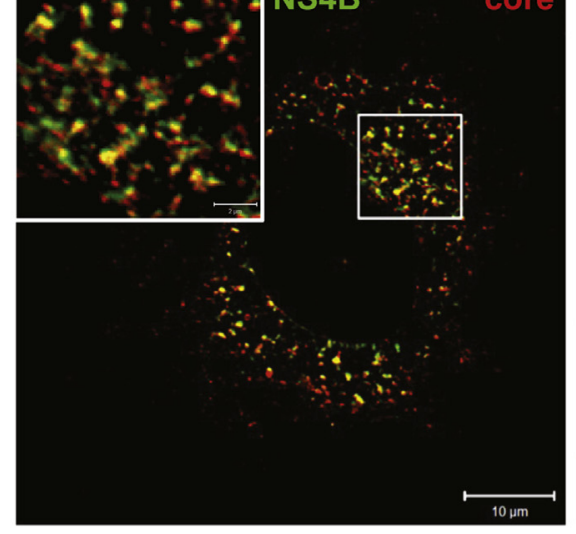

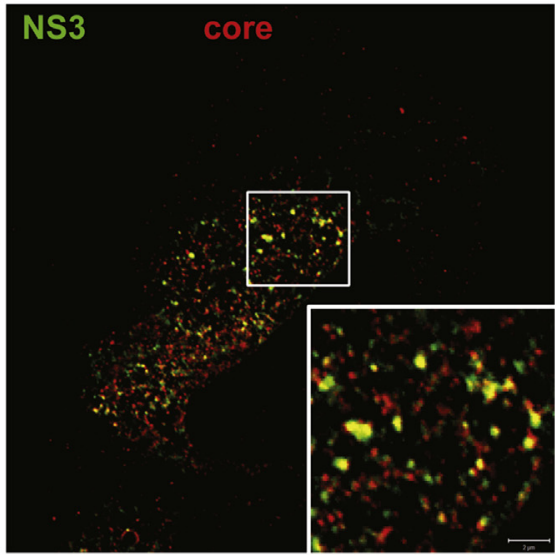

B

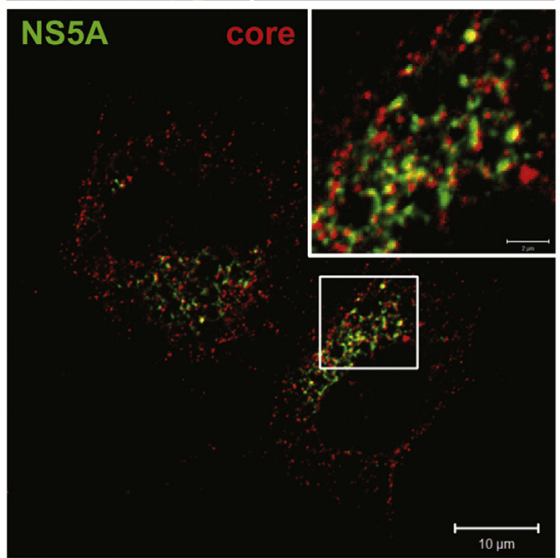

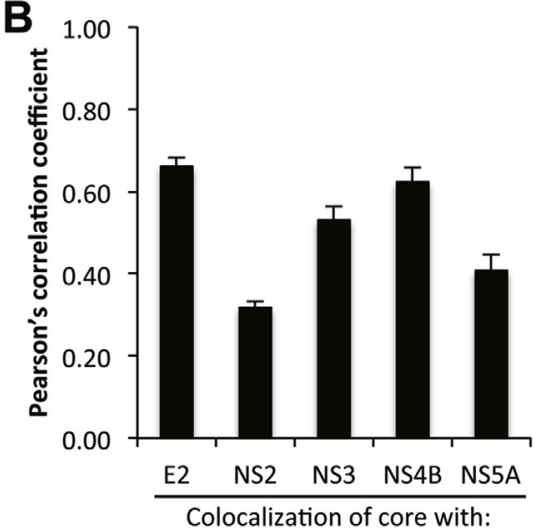

Supplementary Figure 3. Jc1 core punctate structures colocalize with E2 and elements of the replication complexes. Huh7.5 cells were infected with $\mathrm{Jc} 1$ virus at an $\mathrm{MOI}$ of 0.2 , treated for 24 hours with $20 \mu \mathrm{g} / \mathrm{mL} \mathrm{CHX}$ at 48 hours post-infection, and fixed at 72 hours post-infection. Cells were stained for HCV core and other viral proteins, as indicated. (A) Colocalization of core (red channel) with E2, NS2, NS3, NS4B, or NS5A (green channel) was analyzed by confocal microscopy. Scale bars of panels and zooms from squared area represent $10 \mu \mathrm{m}$ and $2 \mu \mathrm{m}$, respectively. $(B)$ The relative degree of colocalization of proteins was quantified by determining Pearson's correlation coefficients of 20 cells by using the JACoP plugin of ImageJ. 
A
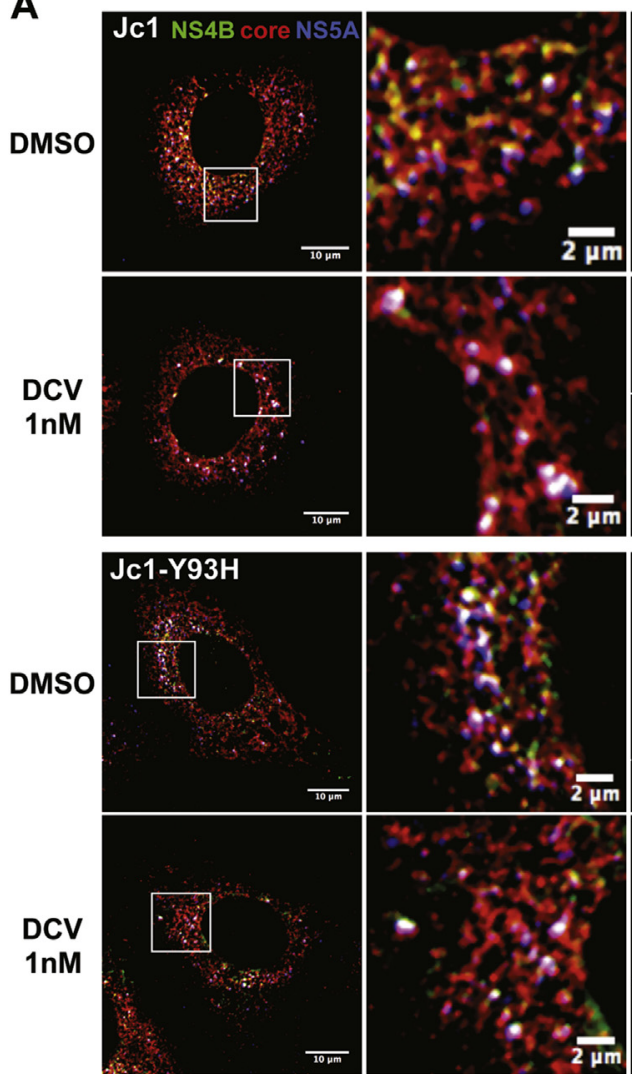
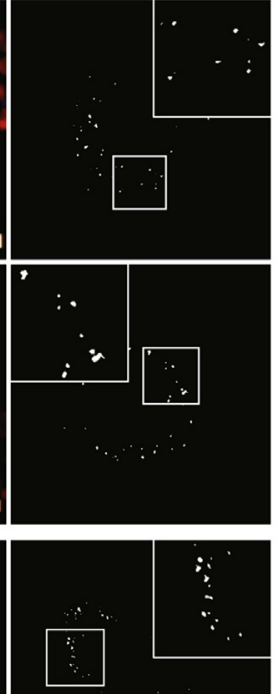

ym

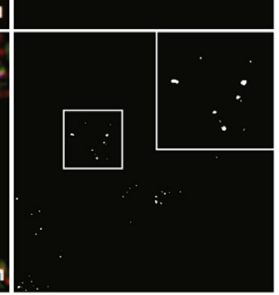

\section{B Surface of structures}
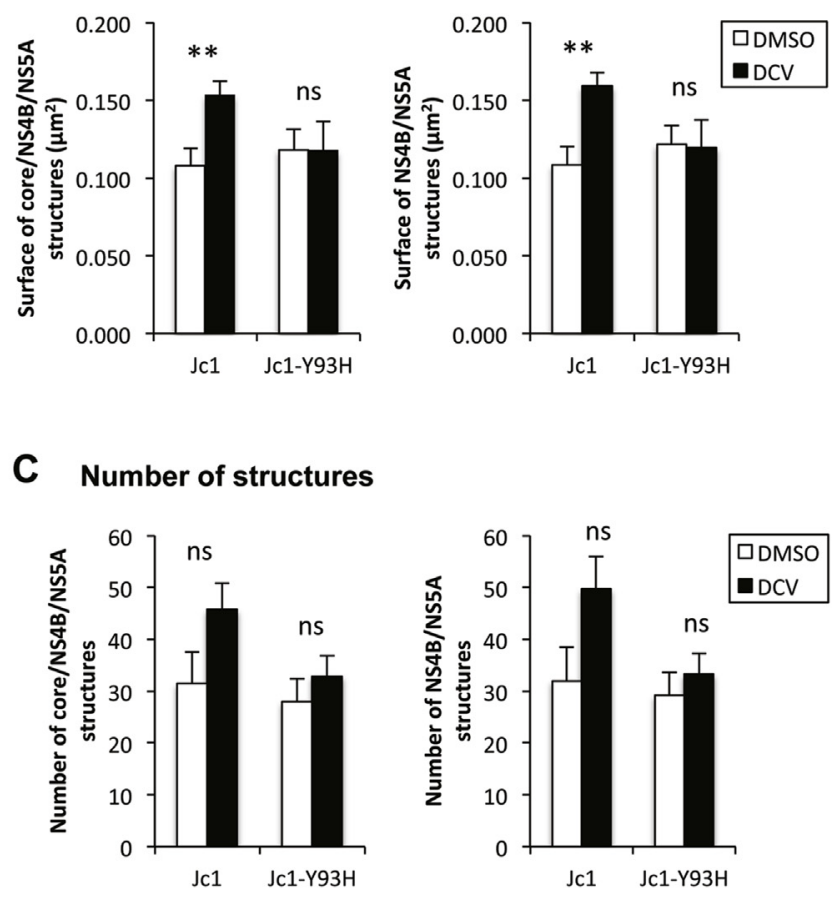

Supplementary Figure 4. DCV induces the clustering of core/NS4B/NS5A structures. Huh7.5 cells infected with Jc1 or Jc1Y93H viruses $(\mathrm{MOI}=0.2)$ were incubated at 66 hours post-infection with $1 \mathrm{nM} \mathrm{DCV}$ or dimethyl sulfoxide (DMSO) for 6 hours. (A) Cells were then stained for HCV core, NS4B, and NS5A proteins and the colocalization of core (red channel) with NS4B (green channel) and NS5A (blue channel) proteins were analyzed by confocal microscopy. Scale bars of panels and zooms from squared area represent $10 \mu \mathrm{m}$ and $2 \mu \mathrm{m}$, respectively. Colocalized pixels (white channel) between red, green, and blue channels were extracted with the ColocalizeRGB plugin of ImageJ. $(B)$ The surface and $(C)$ number of core/NS4B/NS5A and NS4B/NS5A structures were quantified with ImageJ. For each condition, $30-50$ cells were quantified. ${ }^{\star \star \star \star} P \leq .0001$; ${ }^{\star \star \star} P \leq .001 ;{ }^{\star \star} P \leq .01 ;{ }^{\star} P \leq .05$; ns (not significant) $P>.05$. 
A

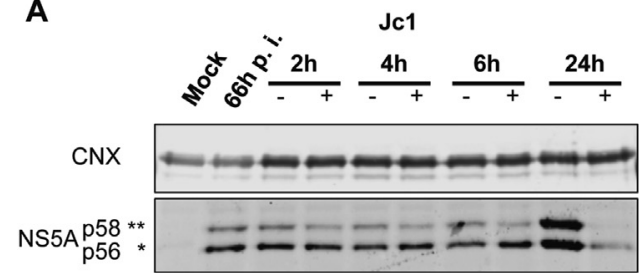

$\begin{array}{llllllll}100 & 74 & 100 & 59 & 100 & 33 & 100 & 7\end{array}$

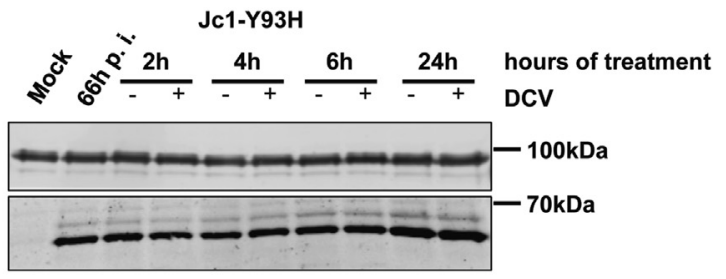

$10014010098 \quad 10095 \quad 100 \quad 84$ Percent of p58

B

Percentage of $\mathrm{p} 58 /$ total NS5A following DCV treatment

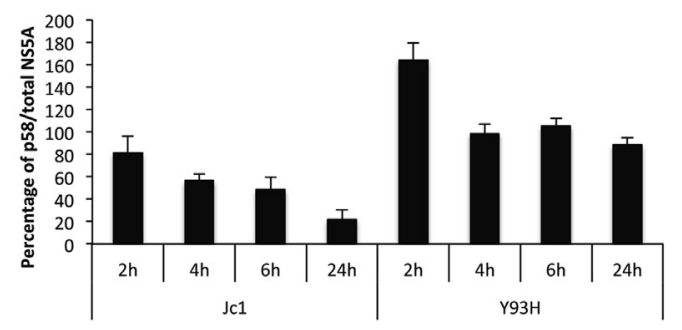

Supplementary Figure 5. Analysis of phosphorylated forms of NS5A. Huh7.5 cells were infected with Jc1 or Jc1-Y93H viruses at an $\mathrm{MOI}$ of 0.2. At 66 hours post-infection, cells were washed and incubated with $1 \mathrm{nM} \mathrm{DCV}$ or dimethyl sulfoxide (DMSO) for the indicated time. Infected cells were harvested at 2, 4, 6, or 24 hours after initiation of DCV treatment and expression of calnexin (CNX) and NS5A proteins was analyzed by Western blot. (A) A representative experiment is shown. The lower $\left(^{*}\right)$ and upper (*) NS5A bands correspond to basal (p56) and hyperphosphorylated (p58) forms of NS5A, respectively. The intensity of each band was quantified by densitometry with ImageJ. The ratio of p58/total NS5A normalized to the internal control calnexin (CNX) (designated \% p58) is indicated below each lane. The values determined for mock-treated samples at each time point were set to $100 \%$. (B) The means of the ratio of p58/total NS5A after DCV treatment from 3 independent experiments are shown. 
A

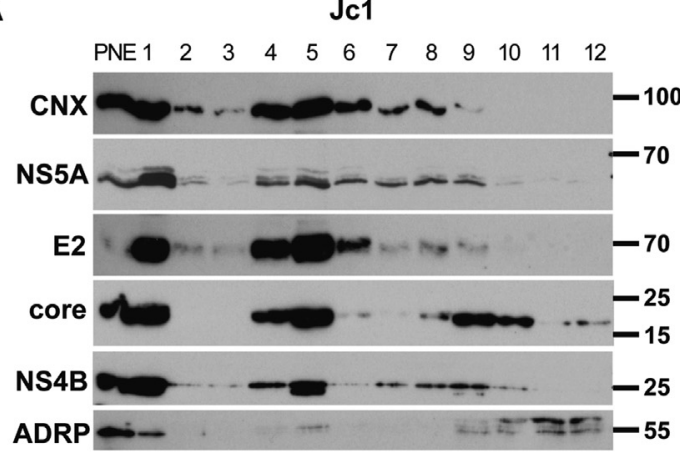

B

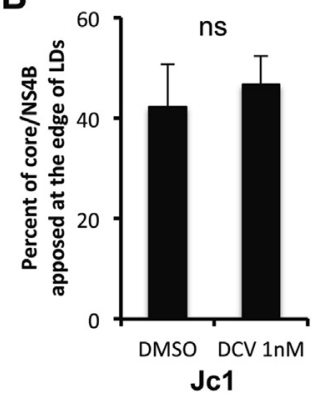

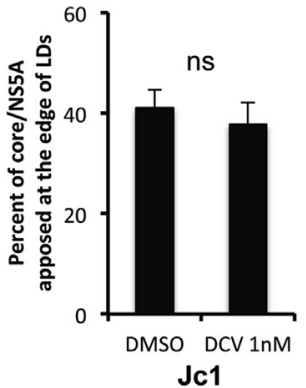

$\mathrm{Jc1}+\mathrm{DCV}$ 1nM

$\begin{array}{llllllllllll}\text { PNE } 1 & 2 & 3 & 4 & 5 & 6 & 7 & 8 & 9 & 10 & 11 & 12\end{array}$
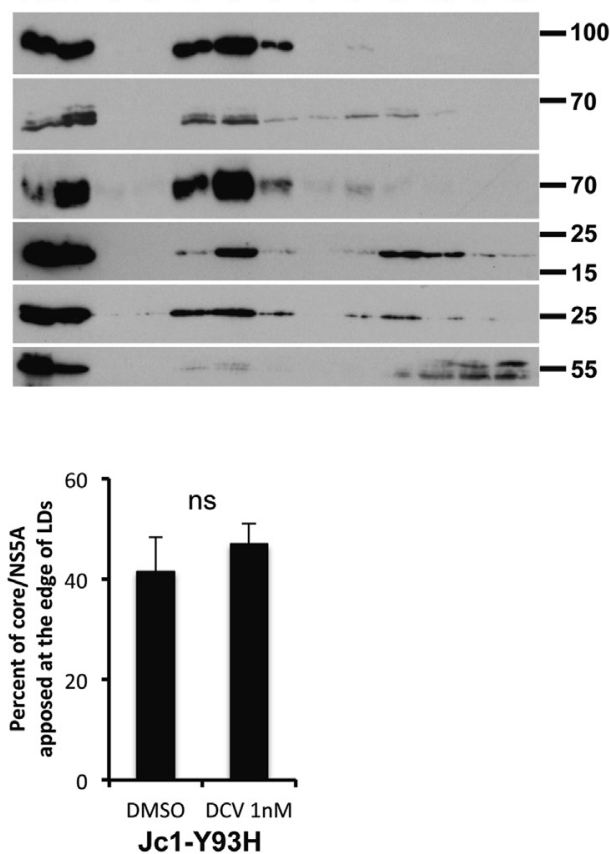

Supplementary Figure 6. Analysis of subcellular distribution of HCV proteins in Jc1-infected cells upon DCV treatment. (A) Huh7.5 cells transfected with Jc1 full-length genome RNA were incubated with $1 \mathrm{nM}$ DCV or dimethyl sulfoxide (DMSO) at 66 hours post-transfection. Transfected cells were lysed at 6 hours after initiation of DCV treatment and were fractionated on iodixanol gradients. Each fraction was analyzed by Western blotting using antibodies against calnexin (CNX), HCV NS5A, E2, core and NS4B proteins, and adipose differentiation-related protein (ADRP). One-fiftieth of unfractionated post-nuclear extracts (PNE) were also analyzed. Results are representative of 5 independent experiments. (B) Huh7.5 cells were infected with Jc1 (lefts panels) or Jc1-Y93H (right panel) viruses at an MOI of 0.2. At 66 hours post-infection, infected cells were washed and incubated with $1 \mathrm{nM}$ of DCV or DMSO. After 6 hours of DCV treatment, cells were fixed and then stained for HCV core, NS5A, and NS4B proteins and for LDs. Colocalization of core and NS4B or core and NS5A proteins with LDs was analyzed by confocal microscopy. The percentage of core/NS4B (left panel) or core/NS5A punctate structures apposed at the edges of LDs in Jc1 (middle panel) or Jc1-Y93H (right panel) infected cells were quantified with ImageJ. ns (not significant), $P>.05$. 


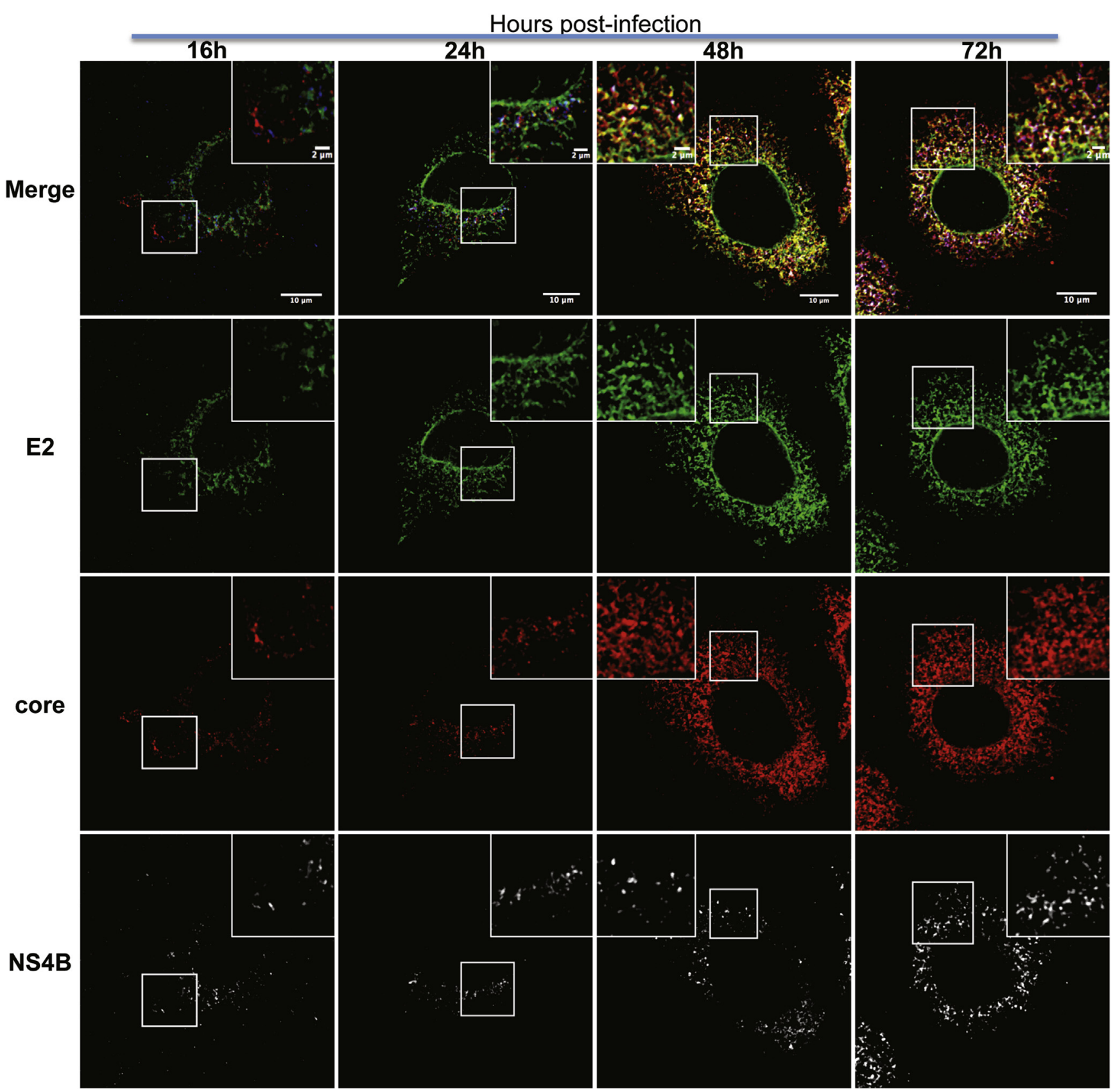

Supplementary Figure 7. Structural proteins and NS4B colocalize within discrete structures. Huh7.5 cells were infected with $\mathrm{Jc} 1$ at an $\mathrm{MOI}$ of 0.2 and were fixed at the indicated times post-infection. Cells were then stained for HCV E2, core, and NS4B proteins and the colocalization of core proteins with E2 and NS4B was analyzed by confocal microscopy. The merge of the 3 channels, as well as individual channels for E2, core, and NS4B proteins (green, red, and white channel, respectively) are represented. Scale bars of panels and zooms from squared area represent $10 \mu \mathrm{m}$ and $2 \mu \mathrm{m}$, respectively. 
A Hours post-infection

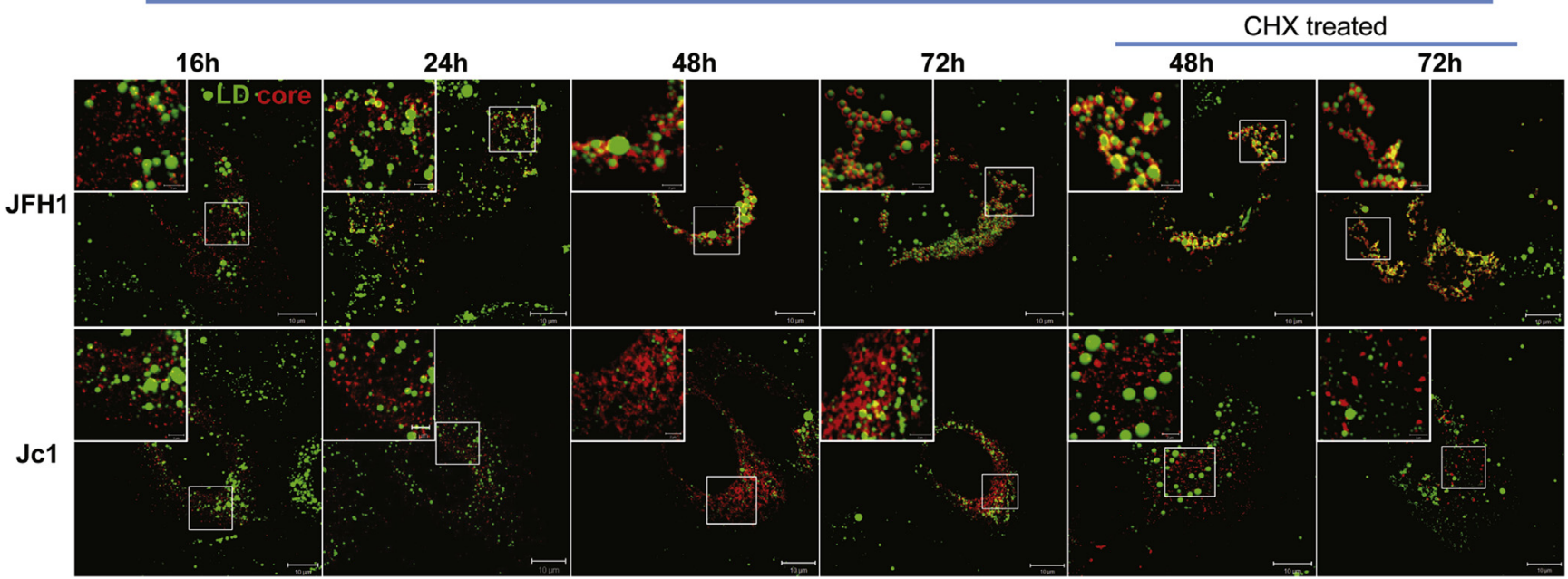

B

C
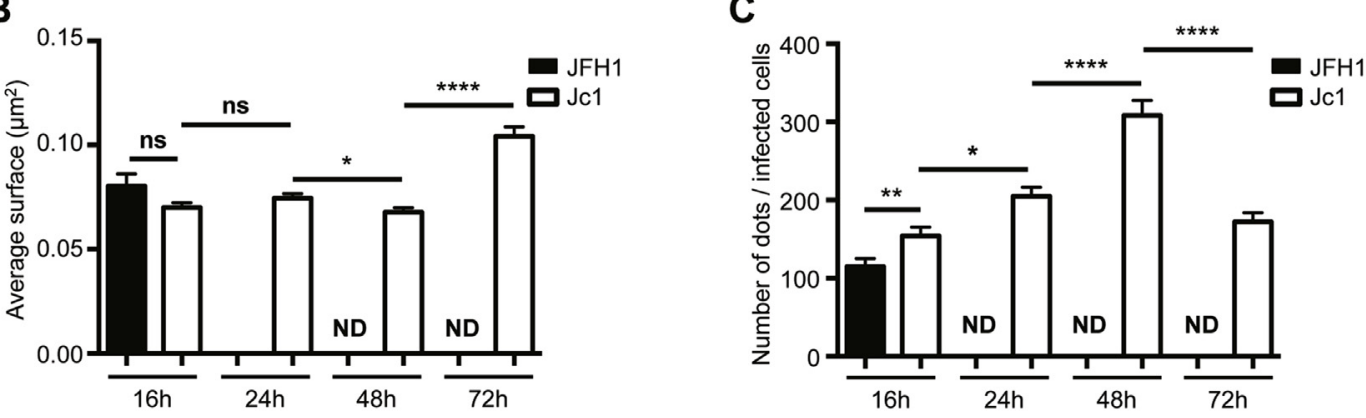

Supplementary Figure 8. Kinetics of HCV core distribution within infected cells. Huh7.5 cells were infected with JFH1 or Jc1 viruses at an $\mathrm{MOI}$ of 0.2 and cultured for the indicated time points with or without a 24-hour treatment with $20 \mu \mathrm{g} / \mathrm{mL} \mathrm{CHX}$ from 24 hours or from 48 hours post-infection, as indicated. Cells were fixed at 16, 24, 48, or 72 hours post-infection, as indicated, and then stained for HCV core and LDs. (A) Colocalization of core protein (red channel) with LDs (green channel) was analyzed by confocal microscopy. Scale bars of panels and zooms from squared area represent $10 \mu \mathrm{m}$ and $2 \mu \mathrm{m}$, respectively. $(B)$ The size and $(C)$ the number of core punctate structures in $\mathrm{CHX}$-treated cells were quantified with ImageJ. For each condition, $30-50$ cells were quantified. ${ }^{\star \star \star *} P \leq .0001$; ${ }^{\star \star \star} P \leq .001 ;{ }^{\star \star} P \leq .01 ;{ }^{*} P \leq .05$; ns (not significant) $P>.05$. ND, not determined. 


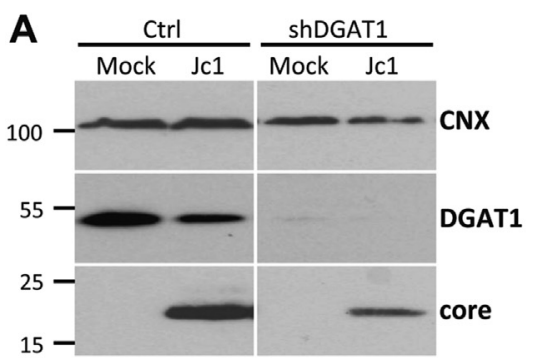

B

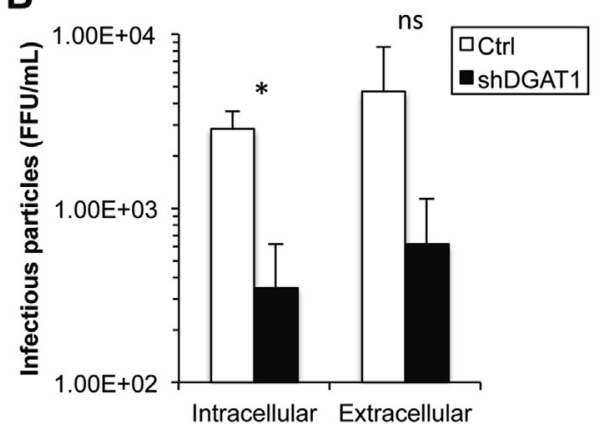

D

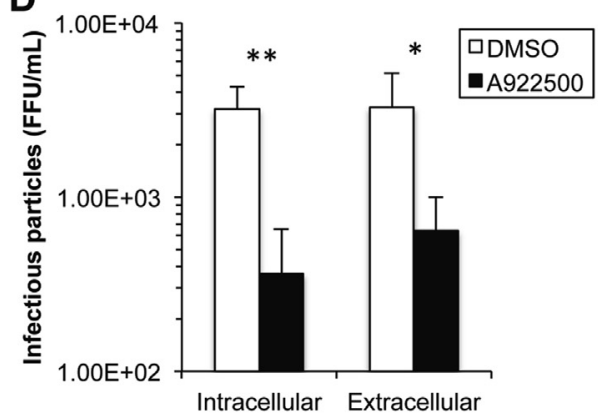

C
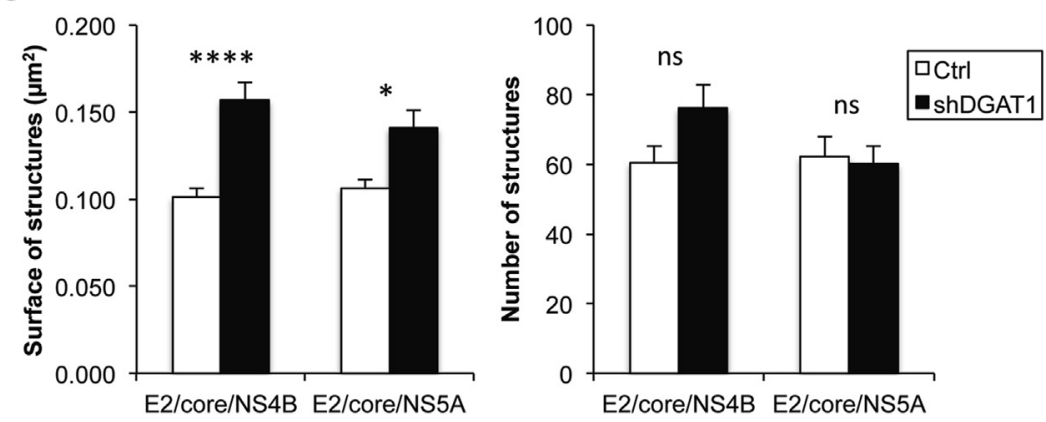

E

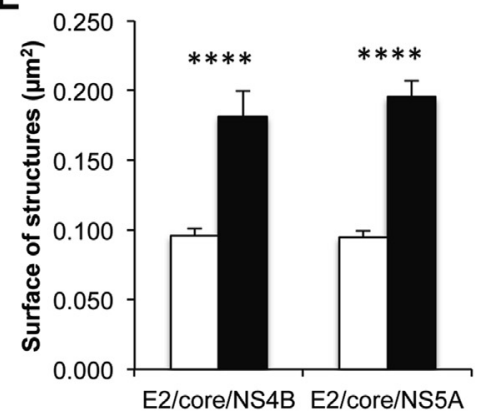

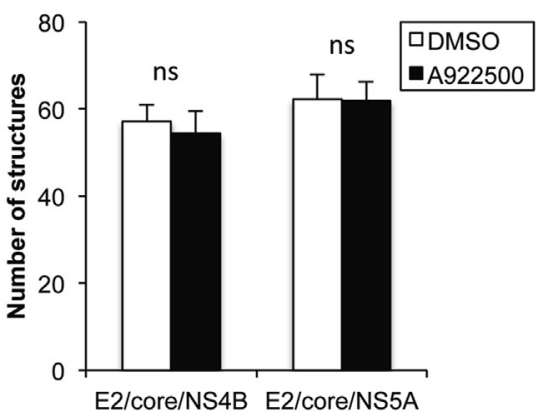

Supplementary Figure 9. DGAT1 down-regulation or inhibition induces the clustering of E2/core/NS4B and E2/core/NS5A structures. Huh7.5 cells were transduced with pLKO.1-puro empty vector (Ctrl) or with pLKO.1-puro vector with DGAT1 shorthairpin RNA (shDGAT1). Seven days post-transduction, down-regulated cells were infected with Jc1 virus at an MOI of 0.2. In separate experiments, Huh7.5 cells were infected with Jc1 virus at an MOI of 0.2 and treated with $150 \mu \mathrm{M}$ of the DGAT1 inhibitor A922500 or dimethyl sulfoxide (DMSO). In both conditions, cells were fixed at 72 hours post-infection, stained for HCV E2, core and NS4B or NS5A proteins and the colocalization of core protein with E2 and NS4B or with E2 and NS5A was analyzed by confocal microscopy. (A) Expression of DGAT1 and calnexin (CNX) and core proteins at 72 hours post-infection was analyzed by Western blot. $(B)$ The intracellular or extracellular infectivity were determined from cell lysates or supernatants of infected cells, respectively. (C) The surface (left panel) and the number (right panel) of E2/core/NS4B and E2/core/NS5A structures were quantified with ImageJ. $(D)$ The intracellular or extracellular infectivity were determined from cell lysates or supernatants of infected cells, respectively. (E) The surface (left panel) and the number (right panel) of E2/core/NS4B and E2/ core/NS5A structures were quantified with ImageJ. For each condition, 20 cells were quantified. ${ }^{\star \star \star \star} P \leq .0001 ;{ }^{\star \star \star} P \leq .001$; ${ }^{*} P \leq .01 ;{ }^{\star} P \leq .05$; ns (not significant) $P>.05$. 